typeset using JPSJ.sty $<$ ver.1.0b $>$

\title{
Pseudogap Phenomena and Superconducting Fluctuations in Hubbard Model for High- $T_{\mathrm{c}}$ Cuprates
}

\author{
Youichi YANASE**** and Kosaku YAMADA \\ Department of Physics, Kyoto University, Kyoto 606-8502
}

(Received October 2, 2000)

\begin{abstract}
The pseudogap phenomena in High- $T_{\mathrm{c}}$ cuprates are investigated on the basis of the Hubbard model which includes only the on-site repulsive interaction $U$. We consider the pairing scenario for the pseudogap. The pseudogap arises from the resonance scattering due to the strong superconducting fluctuations. First, the electronic state and the anti-ferromagnetic spin fluctuations are calculated by using the fluctuation exchange (FLEX) approximation. The T-matrix which is the propagator of the superconducting fluctuations is calculated by extending the Éliashberg equation. The characteristics of the superconducting fluctuations due to the pairing interaction arising from the spin fluctuations are represented in the T-matrix. The self-energy due to the superconducting fluctuations is calculated by the T-matrix approximation. The pseudogap is shown in the single particle properties and the magnetic properties. Moreover, a comprehensive explanation of the doping dependence of the pseudogap is obtained. Furthermore, we apply the theory to the electron-doped cuprates and obtain the consistent results with the recent experiments. Finally, the self-consistent calculation for the spin fluctuations, superconducting fluctuations and the single particle properties are carried out within the FLEX and the self-consistent T-matrix approximations. The relation between the superconducting fluctuations and the spin fluctuations are clarified. The calculated superconducting critical temperature $T_{\mathrm{c}}$ is remarkably reduced from the results of the mean field (FLEX) calculation. In particular, it is shown that the critical temperature decreases with decreasing doping in the under-doped region with large $U$.
\end{abstract}

KEYWORDS: High- $T_{\mathrm{c}}$ cuprates; Hubbard model; Pseudogap; Strong coupling superconductivity; Superconducting fluctuation; spin fluctuation

\section{$\S 1$. Introduction}

The pseudogap phenomena in under-doped High- $T_{\mathrm{c}}$ cuprates have been interested for many years. The pseudogap is considered to be a key issue for the comprehensive understanding of the High- $T_{\mathrm{c}}$ superconductivity.

The pseudogap phenomena mean the suppression of the spectral weight above $T_{\mathrm{c}}$ without any long range order. They are universal phenomena observed in various compounds of High- $T_{\mathrm{c}}$ cuprates in the under-doped region. First, the pseudogap was found in the magnetic excitation channel by the nuclear magnetic resonance (NMR) experiment 1 ) At present, the pseudogap phenomena have been observed in various quantities which include NMR, 1.6 , 3,0 , 6, neutron scattering 8 transport, 2 (10) optical spectrum, 11 electronic specific heat, 12 density of states 13 ) and the single particle spectral weight.44 The experimental results are reviewed in ref. 15 .

There are many theoretical scenarios proposed for the pseudogap phenomena. An important one is the resonating valence bond (RVB) theory. The RVB theory is an approach from the non-Fermi liquid state and attributes the pseudogap to the singlet pairing of the spinons. 16 As an approach from the Fermi liquid state, the magnetic

* E-mail: yanase@hosi.phys.s.u-tokyo.ac.jp

** Present address: Department of Physics, University of Tokyo, Tokyo 113-0033 scenario has been investigated.17.18) In this scenario, the pseudogap is an anti-ferromagnetic gap formation or its precursor.

In this paper, we derive the pseudogap phenomena which arise from the strong superconducting fluctuations. The strong superconducting fluctuations originates from the strong coupling superconductivity and the quasi-two dimensionality 19 ) This theory belongs to the pairing scenario in which the pseudogap is a precursor of the superconductivity. Some aspects of the observed pseudogap have supported the pairing scenario. In particular, the measurements of the single particle properties have given the important features of the pseudogap. The same energy scale and the same momentum dependence between the pseudogap and the superconducting gap have been shown by the angle-resolved photo-emission spectroscopy (ARPES).14) The tunneling spectroscopy have also shown the smooth change from the pseudogap state to the superconducting state.13) The close relation between the pseudogap state and the superconducting state clearly indicates that the pseudogap is a precursor of the superconductivity.

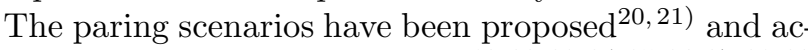
tively investigated in recent years $19.22,23,24,25,26,27,28,29,30,31,32,33,34$, They are classified into some kinds. The scenario based on the phase fuctuations has been proposed by Emery and Kivelson 21) and calculated by other authors.22, 23 The strong phase fluctuations in the ordered state are expected in the under-doped region since the London 
penetration depth $\lambda$ is long 43) The small London constant $\Lambda=1 / 4 \pi \lambda^{2}$ means the small phase stiffness.

The importance of the strong coupling supercenductivity has been pointed out by Randeria et a 20 on the hasis of the Nozières and Schmitt-Rink (NSR) theory.44,45) The NSR theory describes the crossover from the conventional BCS superconductivity to the Bose condensation of tightly-bound pre-formed pairs. The concept of the NSR theory is that the crossover is described by only shifting the chemical potential $\mu$. In case of the strong attractive interaction, the fermions form the pre-formed pairs without phase coherence. In this case, the gap opens in the spectrum of the fermions. The low energy excitations correspond to the pre-formed bosons. The NSR theory has been investigated by many authors because the gap in the fermignicexcitations is considered to be the pseudogap. 20, 24, 25, 26, 27) The existence of the pre-formed pairs has been proposed phenomenologically by Geshkenbein et al.28 with reference to the sign problem of the fluctuational Hall effect.46.

The strength of the superconducting coupling is indicated by the ratio $T_{\mathrm{c}}^{\mathrm{MF}} / \varepsilon_{\mathrm{F}}$. Here, $\varepsilon_{\mathrm{F}}$ is the effective Fermi energy, and $T_{\mathrm{c}}^{\mathrm{MF}}$ is the superconducting critical temperature obtained by the mean field theory. Because the assumption of the weak coupling $T_{\mathrm{c}}^{\mathrm{MF}} / \varepsilon_{\mathrm{F}} \ll 1$ is justified in usual superconductors, the BCS mean field theory correctly describes the superconducting phase transition. The strong coupling superconductivity means that the assumption is violated and therefore the superconducting fluctuations play an important role. The effects of the fluctuations are furthermore strong in the quasitwo dimensional systems. The small $\varepsilon_{\mathrm{F}}$, high- $T_{\mathrm{c}}$ and the quasi-two dimensionality are the characteristics of the cuprates. Therefore, it is natural to consider the strong superconducting fluctuations in High- $T_{\mathrm{c}}$ cuprates.

The NSR theory takes into account the correction of the superconducting fluctuations on the chemical potential $\mu$. However, we have asserted that the NSR theory is justified in the low density limit and not in High- $T_{\mathrm{c}}$ cuprates which are high density systems.19) Therefore, the NSR scenario should be ruled out as a candidate of the pseudogap phenomena.

In the high density systems, the effect of the strong superconducting fluctuations manifests in a different way. It has been shown that the pseudogap phenomena are derived by the self-energy correction due to the strong superconducting fluctuations 19,290 The resonance scattering by the low energy superconducting fluctuations gives rise to the anomalous features of the self-energy and leads to the pseudogap. These effects have been neglected in the NSR theory. The large weight of the superconducting fluctuations at the low energy necessarily exists when the strong coupling supercanductivity occurs in the quasi-two dimensional systems 19,31) We adopt the resonance scattering scenario also in this paper.

We furthermore emphasize that our scenario is clearly differentiated from the NSR scenario. The phase transition in the NSR theory is regarded as the Bose condensation of the pre-formed pairs. However, the phase transition is the superconductivity with strong fluctuations in our scenario.32 33 The later is the realistic situation in High- $T_{\mathrm{c}}$ cuprates.

The effects of the resonance scattering are especially weak in the usual weak coupling superconductors. In these cases, the self-energy correction has not been emphasized in the theory of the superconducting fluctuations, since they are less singular compared with the correction on the two-body correlation function, such as the AL term and the MT term.47.48) However, the superconducting fluctuations have strong effects on the electronic state in the strong coupling case.

The resonance scattering scenario properly explains the magnetic field dependences of the pseudogap phenomena measured by the high field NMR experiments.49, 50,51,52 The calculation gives a comprehensive explanation of the experimental results including their doping dependence 34) Moreover, We have explained the pseudogap phenomena in various experiments such as ARPES, tunneling spectroscopy, NMR, in-plane and $c$ axis transport and the optical conductivity. 33

Thus, the pseudogap phenomena are well explained on the basis of the resonance scattering scenario. However, most of the calculations are based on the model with an attractive interaction. Our previous calculations are also based on the effective model in which the effectively strong $d$-wave pairing interaction affects the renormalized quasi-particles.19, 31, 32, 33, 34) Actually, the $d$-wave pairing interaction arises from the repulsive interaction between the electrons.

Here, it is one of the most important issues to describe the microscopic theory which starts from the repulsive interaction and derives the superconducting fluctuations and the pseudogap phenomena. It should be confirmed that the superconducting coupling is strong enough to lead to the pseudogap phenomena in the under-doped region. Such microscopic calculation will naturally reproduce the doping dependence of the pseudogap phenomena. As is mentioned before,19) there are two important factors in order to realize the strong coupling superconductivity. One is that the effective Fermi energy $\varepsilon_{\mathrm{F}}$ is renormalized by the electron-electron correlation. The other is that the high critical temperature $T_{\mathrm{c}}$ is obtained by the pairing interaction mediated by the anti-ferromagnetic spin fluctuations.53.54) The spin fluctuations also result from the strong electron-electron correlation. Thus, the both factors are derived from the electron-electron correlation and should be described in a unified way. The main purpose of this paper is describing the microscopic theory by which the superconducting fluctuations and the the pseudogap phenomena are derived from the electron-electron correlation.

Some authors have calculated the pseudogap phenomena bystarting from the model with an repulsive interaction. 37. 39.40) However, the sufficient result describing the pseudogap phenomena has not been obtained without any phenomenology.

In this paper, we start from the Hubbard model which is an typical model for the strongly correlated electron systems. First, we describe the quasi-particles and the anti-ferromagnetic spin fluctuations by using the FLEX approximation. The characteristic momentum dependence arising from the anti-ferromagnetic spin fluctua- 
tions55,56) are described by the FLEX approximation. The superconducting fluctuations are derived from the pairing interaction mediated by the spin fluctuations. The derived superconducting fluctuations include the characteristic momentum and frequency dependence of the spin fluctuations. The self-energy due to the superconducting fluctuations is obtained by the one-loop approximation (T-matrix approximation). As a result, the comprehensive understanding is obtained for the pseudogap phenomena on the basis of the resonance scattering scenario. The doping dependence which includes the electron-doped cuprates is also explained consistently.

This paper is constructed as follows. In $\S 2$, we explain the Hubbard Hamiltonian and the FLEX approximation. In $§ 3.1$, we explain the formalism which describes the superconducting fluctuations arising from the spin fluctuations and the self-energy due to the superconducting fluctuations. The calculated results for the single particle properties are shown in $\S 3.2$. In $\S 3.3$, we show the characteristics of the superconducting fluctuations including their doping dependence. The magnetic properties in the pseudogap state are explained in $§ 3.4$. The consistent results with the NMR and the neutron scattering experiments are obtained. In $\S 3.5$, the theory of the superconductivity and the pseudogap phenomena is applied to the electron-doped cuprates. The relevant results including the particle-hole asymmetry are obtained. In $\S 4.1$, we clarify the relation between the superconducting fluctuations and the spin fluctuations in details. In $\S 4.2$, we show the results of the self-consistent calculation including the spin fluctuations, the superconducting fluctuations and the single particle properties. The critical temperature reduced by the fluctuations are calculated. As a result, the appropriate phase diagram is obtained. In $\S 5$, we summarize the obtained results and gives some discussions.

\section{§2. Hubbard Model and FLEX Approximation}

First, we explain the Hubbard model and the FLEX approximation. The Hubbard model has been used for a long time as one of the typical models describing the strongly correlated electron systems. The Hamiltonian is described as,

$$
H=\sum_{\boldsymbol{k}, s} \varepsilon_{\boldsymbol{k}} c_{\boldsymbol{k}, s}^{\dagger} c_{\boldsymbol{k}, s}+U \sum_{\boldsymbol{k}, \boldsymbol{k}^{\prime}, \boldsymbol{q}} c_{\boldsymbol{q}-\boldsymbol{k}^{\prime}, \downarrow}^{\dagger} c_{\boldsymbol{k}^{\prime}, \uparrow}^{\dagger} c_{\boldsymbol{k}, \uparrow} c_{\boldsymbol{q}-\boldsymbol{k}, \downarrow}
$$

The first term is the kinetic term. In this paper, we adopt the two-dimensional dispersion relation $\boldsymbol{\varepsilon}_{\boldsymbol{k}}$ given by the tight-binding model for a square lattice including the nearest- and next-nearest-neighbor hopping $t, t^{\prime}$, respectively,

$$
\varepsilon_{\boldsymbol{k}}=-2 t\left(\cos k_{x}+\cos k_{y}\right)+4 t^{\prime} \cos k_{x} \cos k_{y}-\mu .
$$

We fix $t=0.5, t^{\prime}=0.25 t$ and the lattice constant $a=1$. In this case, the band width $W=4$. The chemical potential $\mu$ is determined according as the filling $n$. These parameters well reproduce the typical Fermi surface of
High- $T_{\mathrm{c}}$ cuprates (see Fig. 13). We define the holedoping concentration $\delta=1-n$. The main part of this paper is concerned with the hole-doped cuprates $\delta>0$. A brief application to the electron-doped cuprates $\delta<0$ will be carried out in $§ 3.5$. The nearly half-filling systems, which are interested here, should be regarded as high-density systems. Therefore, the shift of the chemical potential, which is considered in the NSR scenario, is not important in this paper. The second term in eq. (2.1) expresses the on-site repulsive interaction between the electrons. All of the phenomena described in this paper result from the electron correlation effects.

The FLEX approximation has been used in order to describe the strongly correlatedelectron systems near the anti-ferromagnetic instability.57) The FLEX approximation is a conserving approximation 58 and describes the anti-ferromagnetic spinfluctuations including the mode coupling effects partly.59,60) It has been shown that the superconducting critical temperature with a relevant order $T_{\mathrm{c}} \sim 100 \mathrm{~K}$ is obtained by the EIEX approximation for High- $T_{\mathrm{c}}$ cuprates.61, 62, 63, 64, 65, 66, 67) The relevant results are also obtained for the proanic superconductor $\kappa$-(BEDT-TTF) compounds 68, 69, 70 and for the ladder type compound $\mathrm{Sr}_{14-x} \mathrm{Ca}_{x} \mathrm{Cu}_{24} \mathrm{O}_{41}$ 71) Moreover, the FLEX approximation is used to explain the anomalous properties resulting from the anti-ferromagnetic spin fluctuations, such as the Hall coefficient 72, 730 the neutron resonance peak and the dip-hump structure in ARPES.74)

The self-energy given by the FLEX approximation $\Sigma_{\mathrm{F}}\left(\boldsymbol{k}, \mathrm{i} \omega_{n}\right)$ is expressed by the one-loop diagram exchanging the normal vertex $V_{\mathrm{n}}\left(\boldsymbol{q}, \mathrm{i} \Omega_{n}\right)$ (Fig. 1(a)).

$$
\Sigma_{\mathrm{F}}\left(\boldsymbol{k}, \mathrm{i} \omega_{n}\right)=T \sum_{\boldsymbol{q}, \mathrm{i} \Omega_{n}} V_{\mathrm{n}}\left(\boldsymbol{q}, \mathrm{i} \Omega_{n}\right) G\left(\boldsymbol{k}-\boldsymbol{q}, \mathrm{i} \omega_{n}-\mathrm{i} \Omega_{n}\right) .
$$

The normal vertex is expressed as follows,

$$
V_{\mathrm{n}}\left(\boldsymbol{q}, \mathrm{i} \Omega_{n}\right)=U^{2}\left[\frac{3}{2} \chi_{\mathrm{s}}\left(\boldsymbol{q}, \mathrm{i} \Omega_{n}\right)+\frac{1}{2} \chi_{\mathrm{c}}\left(\boldsymbol{q}, \mathrm{i} \Omega_{n}\right)-\chi_{0}\left(\boldsymbol{q}, \mathrm{i} \Omega_{n}\right)\right] .
$$

Here, $\chi_{\mathbf{s}}\left(\boldsymbol{q}, \mathrm{i} \Omega_{n}\right)$ and $\chi_{\mathrm{c}}\left(\boldsymbol{q}, \mathrm{i} \Omega_{n}\right)$ represent the spin and charge susceptibility, respectively,

$$
\begin{aligned}
& \chi_{\mathrm{s}}\left(\boldsymbol{q}, \mathrm{i} \Omega_{n}\right)=\frac{\chi_{0}\left(\boldsymbol{q}, \mathrm{i} \Omega_{n}\right)}{1-U \chi_{0}\left(\boldsymbol{q}, \mathrm{i} \Omega_{n}\right)}, \\
& \chi_{\mathrm{c}}\left(\boldsymbol{q}, \mathrm{i} \Omega_{n}\right)=\frac{\chi_{0}\left(\boldsymbol{q}, \mathrm{i} \Omega_{n}\right)}{1+U \chi_{0}\left(\boldsymbol{q}, \mathrm{i} \Omega_{n}\right)},
\end{aligned}
$$

where $\chi_{0}\left(\boldsymbol{q}, \mathrm{i} \Omega_{n}\right)$ is the irreducible susceptibility,

$$
\chi_{0}\left(\boldsymbol{q}, \mathrm{i} \Omega_{n}\right)=-T \sum_{\boldsymbol{k}, \mathrm{i} \omega_{n}} G\left(\boldsymbol{k}, \mathrm{i} \omega_{n}\right) G\left(\boldsymbol{k}+\boldsymbol{q}, \mathrm{i} \omega_{n}+\mathrm{i} \Omega_{n}\right) .
$$

The first order term in $V_{\mathrm{n}}\left(\boldsymbol{q}, \mathrm{i} \Omega_{n}\right)$ with respect to $U$ is eliminated because it gives only the trivial HartreeFock term. In the FLEX approximation, the dressed Green function $G\left(\boldsymbol{k}, \mathrm{i} \omega_{n}\right)=\left(\mathrm{i} \omega_{n}-\varepsilon_{\boldsymbol{k}}-\Sigma_{\mathrm{F}}\left(\boldsymbol{k}, \mathrm{i} \omega_{n}\right)\right)^{-1}$ is used. The self-energy and the spin susceptibility are determined self-consistently. Equations. (2.3-6) are self- 
(a)

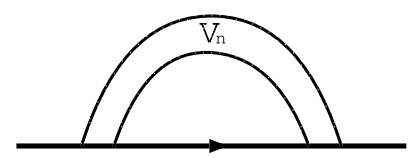

(b)

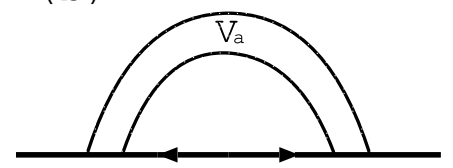

Fig. 1. (a) The normal self-energy and (b) the anomalous selfenergy obtained by the FLEX approximation.

consistently solved by the numerical calculation. In the main part of this paper, we divide the first Brillouin zone into $64 \times 64$ lattice points for the numerical calculation. The spin susceptibility given by the FLEX approximation $\chi_{\mathrm{s}}\left(\boldsymbol{q}, \mathrm{i} \Omega_{n}\right)$ is enhanced near the anti-ferromagnetic wave vector $\boldsymbol{Q}=(\pi, \pi)$. The antiferromagnetic spin fluctuations described by $\chi_{\mathbf{s}}\left(\boldsymbol{q}, i \Omega_{n}\right)$ play a dominant role in the FLEX approximation. The characteristic results of the nearlyanti-ferromagnetic Fermi liquid theory $17,53,54,55,56,59,60,72,73,55$ are qualitatively reproduced within the FLEX approximation.

The superconducting critical temperature $T_{\mathrm{c}}$ is determined as the temperature below which the linearized Dyson-Gor'kov equation has a non-trivial solution (Fig. 1(b)). The criterion for $T_{\mathrm{c}}$ is described by the Éliashberg equation which is the following eigenvalue equation,

$$
\begin{aligned}
\lambda \phi\left(\boldsymbol{k}, \mathrm{i} \omega_{n}\right)=- & T \sum_{\boldsymbol{p}, \mathrm{i} \omega_{m}} V_{\mathrm{a}}\left(\boldsymbol{k}-\boldsymbol{p}, \mathrm{i} \omega_{n}-\mathrm{i} \omega_{m}\right) \\
& \times\left|G\left(\boldsymbol{p}, \mathrm{i} \omega_{m}\right)\right|^{2} \phi\left(\boldsymbol{p}, \mathrm{i} \omega_{m}\right) .
\end{aligned}
$$

Here, $V_{\mathrm{a}}\left(\boldsymbol{q}, \mathrm{i} \Omega_{n}\right)$ is the anomalous vertex for the singlet channel. This is given by the FLEX approximation as follows,

$$
V_{\mathrm{a}}\left(\boldsymbol{q}, \mathrm{i} \Omega_{n}\right)=U^{2}\left[\frac{3}{2} \chi_{\mathrm{s}}\left(\boldsymbol{q}, \mathrm{i} \Omega_{n}\right)-\frac{1}{2} \chi_{\mathrm{c}}\left(\boldsymbol{q}, \mathrm{i} \Omega_{n}\right)\right]+U .
$$

The critical temperature $T_{\mathrm{c}}$ is obtained as the temperature where the maximum eigenvalue $\lambda_{\max }$ becomes the unity $\lambda_{\max }=1$. The eigenfunction $\phi_{\max }\left(\boldsymbol{p}, \mathrm{i} \omega_{m}\right)$ corresponding to the eigenvalue $\lambda_{\max }$ is the wave function of the Cooper pairs. In this paper, the symmetry of the superconductivity is always the $d_{x^{2}-y^{2}}$-wave.

Here, we show the typical results of the FLEX approximation in Figs. 2-4. The results for the analytically continuated self-energy $\Sigma_{\mathrm{F}}^{\mathrm{R}}(\boldsymbol{k}, \omega)$ are shown in Fig. 2. In this paper, the analytic continuation is carried out by using the Padé approximation. The real part $\operatorname{Re} \Sigma_{\mathrm{F}}^{\mathrm{R}}(\boldsymbol{k}, \omega)$ shows the negative slope and the imaginary part $\operatorname{Im} \Sigma_{\mathrm{F}}^{\mathrm{R}}(\boldsymbol{k}, \omega)$ has the minimum absolute value at the Fermi level. They are the characteristic behaviors of the Fermi liquid theory which is violated in the pseudogap state.

Some notable features are present in the self-energy. The imaginary part shows the $\omega$-linear dependence near the Fermi level contrary to the $\omega$-square dependence in the conventional Fermi liquid theory. The behavior has the same origin as that of the $T$-linear resistivity 55,56 The imaginary part at $\omega=0$, that is the quasi-particle's damping, is large near $(\pi, 0)$ ('hot spot') and is small near $(\pi / 2, \pi / 2)$ ('cold spot'). The above momentum dependence plays a crucial role in the coherent in-plane transport and the incoherent $c$-axis transport 33, 55, [6] The renormalization factor $Z_{\boldsymbol{k}}^{-1}=1-\partial \operatorname{Re} \Sigma_{\mathrm{F}}^{\mathrm{R}}(\boldsymbol{k}, \omega) / \partial \omega$ has the qualitatively same feature as the quasi-particle's damping. A lot of the low energy states near $(\pi, 0)$ arise from the Van Hove singularity and the large renormalization factor $Z_{\boldsymbol{k}}^{-1}$. Because the Fermi surface is transformed by the electron correlation, the Van Hove singularity approaches the Fermi level much more. The transformation of the Fermi surface is a characteristic feature of the nearly anti-ferromagnetic systems 56 and is confirmed by the FLEX approximation.22 The effective Fermi energy for the superconducting fluctuations are renormalized by the above features.

The results for the single particle spectral weight and the density of states (DOS) are shown in Fig. 3. In spite of the unconventional Fermi liquid behaviors, the typical single peak structure appears in the spectral weight. Thus, the picture of the quasi-particles holds in the nearly anti-ferromagnetic Fermi liquid. In particular, the pseudogap is not seen. The spectral weight is remarkably broad at the 'hot spot' and sharp at the 'cold spot' reflecting the momentum dependence of the quasiparticle's lifetime (see Fig. 2(b)). The low energy states near $(\pi, 0)$ give the large DOS near the Fermi level. In this paper a small constant damping is added in the selfenergy when calculating the DOS in order to exclude the finite size effect. This treatment has no significant effect on the obtained results.

The obtained results for the superconducting critical temperature $T_{\mathrm{c}}$ are shown in Fig. 4. It is seen that the critical temperature increases with decreasing the doping concentration $\delta$, or increasing the repulsive interaction $U$. In other words, the critical temperature increases with the development of the anti-ferromagnetic spin fluctuations. The critical temperature tends to be saturated in the large $U$ and the small $\delta$ region. It is a common result of the FLEX calculation because the spin fluctuations have not only the pairing effects but also the de-pairing effects.

\section{§3. FLEX+T-matrix Approximation}

Hereafter, we consider the superconducting fluctuations and their effects on the single particle properties and the magnetic properties. In our scenario, the superconducting fluctuations play a dominant role for the pseudogap phenomena. Therefore, we have to describe the superconducting fluctuations derived from the anomalous vertex $V_{\mathrm{a}}\left(\boldsymbol{q}, \mathrm{i} \Omega_{n}\right)$ given by the FLEX approx- 
(a)

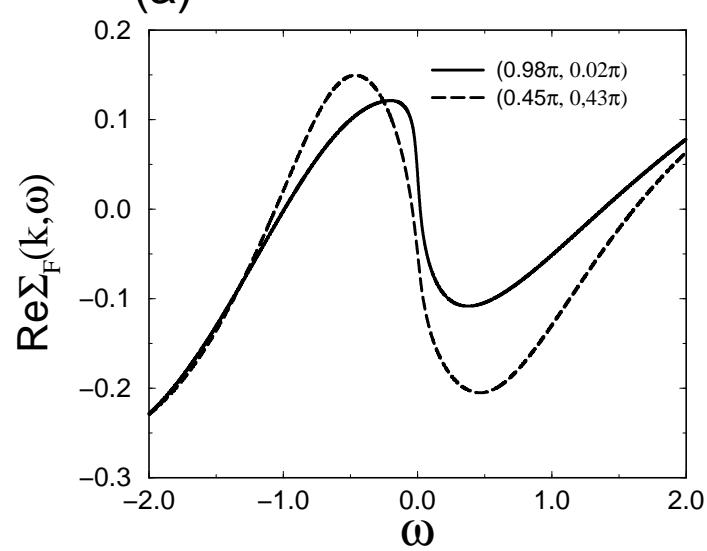

(b)

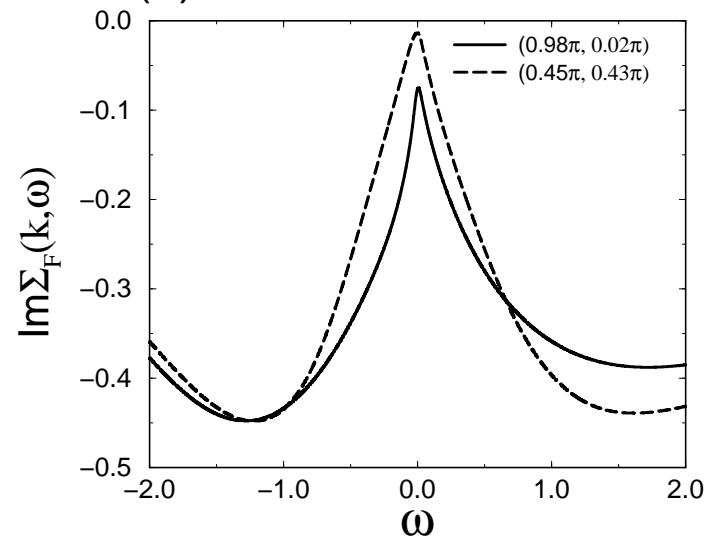

Fig. 2. The self-energy obtained by the FLEX approximation. (a) The real part. (b) The imaginary part. Here, $U=1.6$, $\delta=0.095$ and $T=0.010$. The solid lines and the long-dashed lines correspond to $\left(\frac{63}{64} \pi, \frac{1}{64} \pi\right)$ ('hot spot') and $\left(\frac{29}{64} \pi, \frac{27}{64} \pi\right)$ ('cold spot'), respectively. The value $\operatorname{Re} \Sigma_{\mathrm{F}}^{\mathrm{R}}(\boldsymbol{k}, 0)$ is positive at the 'hot spot' and negative at the 'cold spot'. This means that the Fermi surface is transformed to be more appropriate to the nesting.

imation. Although the calculation has been difficult 39 ) we succeed in the description including the momentum and frequency dependence of the anti-ferromagnetic spin fluctuations. We explain the method of the calculation bellow. The obtained results well explain the pseudogap phenomena including their doping dependence and some characteristic properties. In this section, the coupling constant $U$ is fixed to $U=1.6$ unless we specify.

\subsection{Formalism}

First, we explain the formalism of the FLEX+Tmatrix calculation which includes the effects of superconducting fluctuations within the lowest order. The superconducting fluctuations are generally represented by the T-matrix which is the propagator of the superconducting fluctuations. The T-matrix is expressed by the ladder diagrams in the particle-particle channel (Fig. (a)

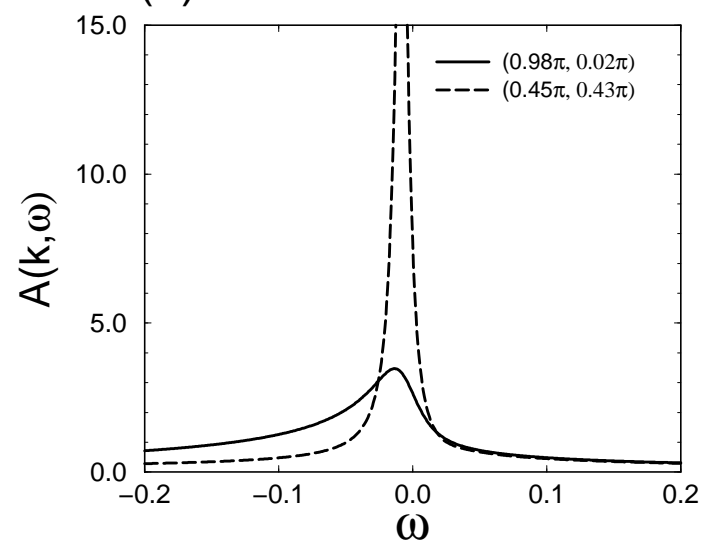

(b)

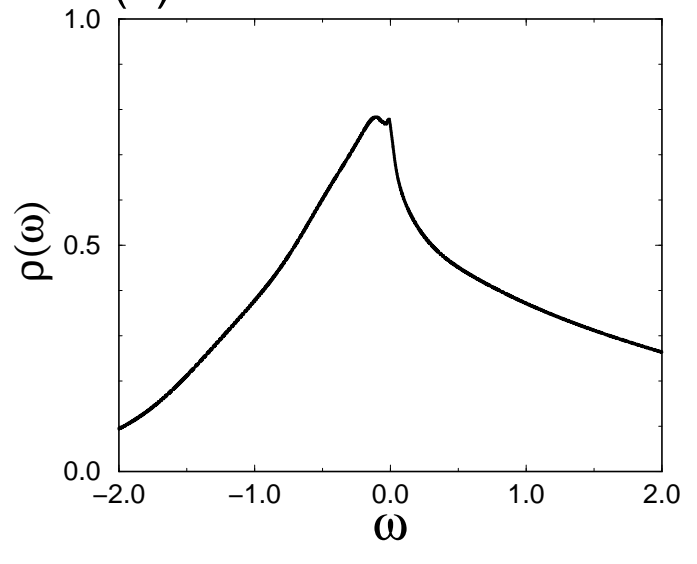

Fig. 3. (a) The single particle spectral weight and (b) the density of states obtained by the FLEX approximation. The parameters are the same as those in the Fig. 2.

5 (a)) and is derived from the following Bethe-Salpeter equation.

$$
\begin{aligned}
& T\left(\boldsymbol{k}_{1}, \mathrm{i} \omega_{n}: \boldsymbol{k}_{2}, \mathrm{i} \omega_{m}: \boldsymbol{q}, \mathrm{i} \Omega_{n}\right)=V_{\mathrm{a}}\left(\boldsymbol{k}_{1}-\boldsymbol{k}_{2}, \mathrm{i} \omega_{n}-\mathrm{i} \omega_{m}\right) \\
& \quad-T \sum_{\boldsymbol{k}, \omega_{l}} V_{\mathrm{a}}\left(\boldsymbol{k}_{1}-\boldsymbol{k}, \mathrm{i} \omega_{n}-\mathrm{i} \omega_{l}\right) G\left(\boldsymbol{k}, \mathrm{i} \omega_{l}\right) \\
& \quad \times G\left(\boldsymbol{q}-\boldsymbol{k}, \mathrm{i} \Omega_{n}-\mathrm{i} \omega_{l}\right) T\left(\boldsymbol{k}, \mathrm{i} \omega_{l}: \boldsymbol{k}_{2}, \mathrm{i} \omega_{m}: \boldsymbol{q}, \mathrm{i} \Omega_{n}\right) .(3.1)
\end{aligned}
$$

Here, the pairing interaction is given by the anomalous vertex obtained by the FLEX approximation. Generally, it is difficult to solve the above integral equation except

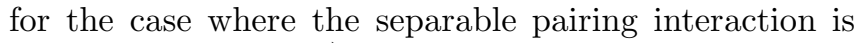
assumed. 19. 31. 32, 33. 34 Therefore, we carry out the following two approximations where the meaningful component as the $d_{x^{2}-y^{2}}$-wave superconducting fluctuations is properly taken out.

The T-matrix at $\boldsymbol{q}=\Omega_{n}=0$ is approximately decomposed into the eigenfunction with its respective eigenvalue of the Éliashberg equation.t0

$$
T\left(\boldsymbol{k}_{1}, \mathrm{i} \omega_{n}: \boldsymbol{k}_{2}, \mathrm{i} \omega_{m}: \boldsymbol{q}=\mathrm{i} \Omega_{n}=0\right)
$$


(a)

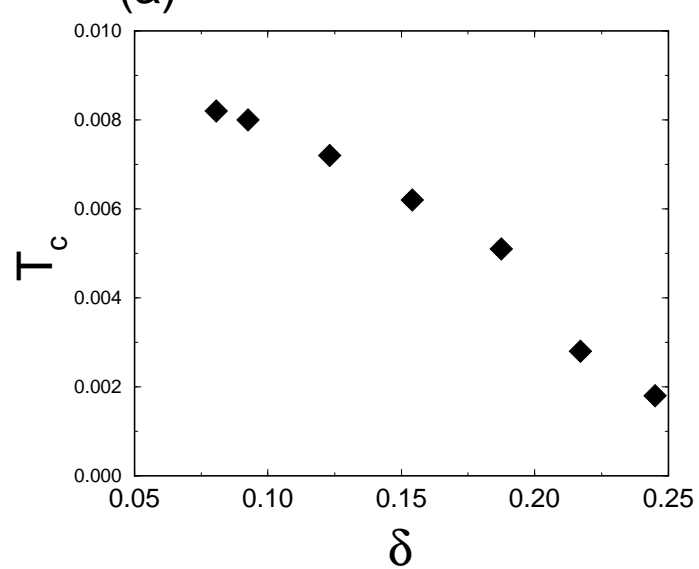

(b)

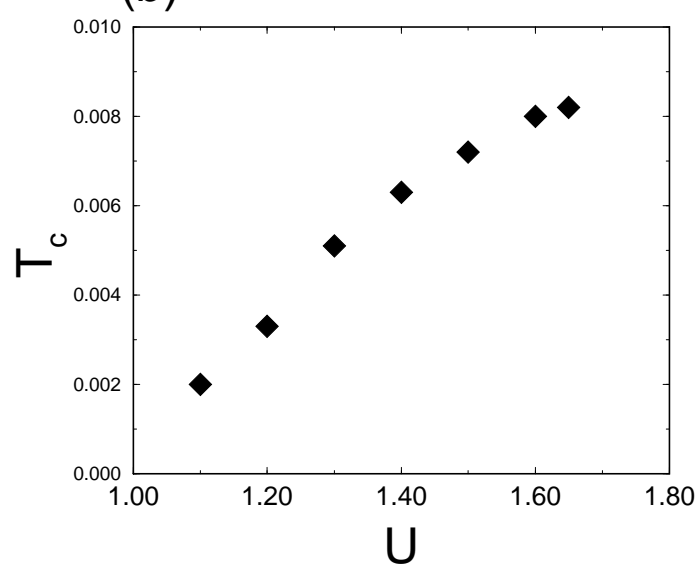

Fig. 4. The superconducting critical temperature obtained by the FLEX approximation. (a) The doping concentration dependence for $U=1.6$. (b) The repulsive interaction dependence for $\delta=$ 0.09 .

(a)

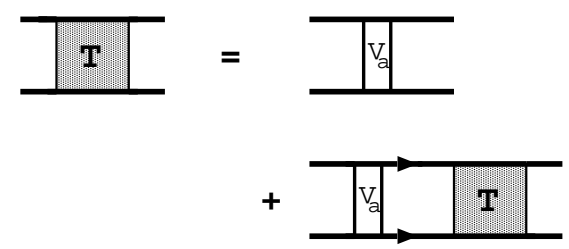

(b)

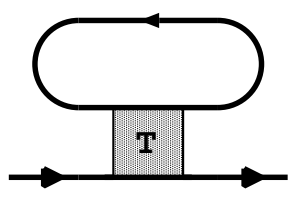

Fig. 5. (a) The T-matrix. (b) The self-energy due to the superconducting fluctuations.

$$
=\sum_{\alpha} \frac{g_{\alpha} \phi_{\alpha}\left(\boldsymbol{k}_{1}, \mathrm{i} \omega_{n}\right) \phi_{\alpha}^{*}\left(\boldsymbol{k}_{2}, \mathrm{i} \omega_{m}\right)}{1-\lambda_{\alpha}} .
$$

The eigenvalue $\lambda_{\alpha}$ and the eigenfunction $\phi_{\alpha}\left(\boldsymbol{k}, \mathrm{i} \omega_{n}\right)$ are derived from the Éliashberg equation, eq. (2.7). The index $\alpha$ denotes each mode included in the T-matrix. Here, we take out the component with the maximum eigenvalue $\lambda_{\max }$ and the corresponding eigenfunction $\phi_{\max }$ which has the $d_{x^{2}-y^{2}}$-wave character. By considering that the superconducting transition is determined by the condition $\lambda_{\max }=1$, we can understand that the mode described by $\lambda_{\max }$ and $\phi_{\max }$ represents the $d_{x^{2}-y^{2-}}$ wave superconducting fluctuations. The function $\phi_{\max }$ is the wave function of the fluctuating Cooper pairs in the fluctuating regime. Hereafter, we neglect the other modes since they have no significant effect on the superconducting fluctuations and the electronic state at the low energy. Hereafter, we neglect the index 'max' for simplicity. The approximation is justified when the superconducting fluctuations are strong. By using the above approximation the T-matrix is expressed by the following equations 39 )

$$
\begin{aligned}
& T\left(\boldsymbol{k}_{1}, \mathrm{i} \omega_{n}: \boldsymbol{k}_{2}, \mathrm{i} \omega_{m}: \boldsymbol{q}, \mathrm{i} \Omega_{n}\right)= \\
& \frac{g\left(\boldsymbol{q}, \mathrm{i} \Omega_{n}\right) \phi\left(\boldsymbol{k}_{1}, \mathrm{i} \omega_{n}: \boldsymbol{q}, \mathrm{i} \Omega_{n}\right) \phi^{*}\left(\boldsymbol{k}_{2}, \mathrm{i} \omega_{m}: \boldsymbol{q}, \mathrm{i} \Omega_{n}\right)}{1-\lambda\left(\boldsymbol{q}, \mathrm{i} \Omega_{n}\right)} .
\end{aligned}
$$

The function $\lambda\left(\boldsymbol{q}, \mathrm{i} \Omega_{n}\right)$ is given by the maximum eigenvalue and $\phi\left(\boldsymbol{k}, \mathrm{i} \omega_{n}: \boldsymbol{q}, \mathrm{i} \Omega_{n}\right)$ is the corresponding eigenfunction of the following equation,

$$
\begin{aligned}
& \lambda\left(\boldsymbol{q}, \mathrm{i} \Omega_{n}\right) \phi\left(\boldsymbol{k}, \mathrm{i} \omega_{n}: \boldsymbol{q}, \mathrm{i} \Omega_{n}\right)=-T \sum_{\boldsymbol{p}, \mathrm{i} \omega_{m}} V_{\mathrm{a}}\left(\boldsymbol{k}-\boldsymbol{p}, \mathrm{i} \omega_{n}-\mathrm{i} \omega_{m}\right) \\
& \times G\left(\boldsymbol{p}, \mathrm{i} \omega_{m}\right) G\left(\boldsymbol{q}-\boldsymbol{p}, \mathrm{i} \Omega_{n}-\mathrm{i} \omega_{m}\right) \phi\left(\boldsymbol{p}, \mathrm{i} \omega_{m}: \boldsymbol{q}, \mathrm{i} \Omega_{n}\right) .
\end{aligned}
$$

The function $1-\lambda\left(\boldsymbol{q}, \mathrm{i} \Omega_{n}\right)$ describes the dispersion relation of the fluctuating Cooper pairs. In the above expression, the wave function is normalized as, $\quad \sum_{\boldsymbol{k}, \mathrm{i} \omega_{n}}\left|\phi\left(\boldsymbol{k}, \mathrm{i} \omega_{n}: \boldsymbol{q}, \mathrm{i} \Omega_{n}\right)\right|^{2}=1$, and the coupling constant is given as, $g\left(\boldsymbol{q}, \mathrm{i} \Omega_{n}\right)=$ $\sum_{\boldsymbol{k}_{1}, \mathrm{i} \omega_{n}} \sum_{\boldsymbol{k}_{2}, \mathrm{i} \omega_{m}} \phi^{*}\left(\boldsymbol{k}_{1}, \mathrm{i} \omega_{n}: \boldsymbol{q}, \mathrm{i} \Omega_{n}\right) V_{\mathrm{a}}\left(\boldsymbol{k}_{1}-\boldsymbol{k}_{2}, \mathrm{i} \omega_{n}-\right.$ $\left.\mathrm{i} \omega_{m}\right) \phi\left(\boldsymbol{k}_{2}, \mathrm{i} \omega_{m}: \boldsymbol{q}, \mathrm{i} \Omega_{n}\right)$.

Here, we perform one more approximation, which is valid around $q=\Omega_{n}=0$. We neglect the $q$ - and $\Omega_{n}$ dependence of the eigenfunction $\phi\left(\boldsymbol{k}, \mathrm{i} \omega_{n}: \boldsymbol{q}, \mathrm{i} \Omega_{n}\right)$. The wave function is fixed to that at $q=\Omega_{n}=0$. This approximation is well justified in the following way. The main $q$ - and $\Omega_{n}$ dependence of the eigenvalue $\lambda\left(\boldsymbol{q}, \mathrm{i} \Omega_{n}\right)$ is derived from the differential of the Green function $G\left(\boldsymbol{q}-\boldsymbol{p}, \mathrm{i} \Omega_{n}-\mathrm{i} \omega_{m}\right)$ in eq. (3.4). In other words, the dispersion of the fluctuating Cooper pairs, $1-\lambda\left(\boldsymbol{q}, i \Omega_{n}\right)$ is insensitive to the change of the eigenfunction. It should be noticed that the wave function $\phi\left(\boldsymbol{k}, \mathrm{i} \omega_{n}: \boldsymbol{q}, \mathrm{i} \Omega_{n}\right)$ is independent of $q$ and $\Omega_{\text {m }}$ when the separable paring interaction is considered.19)

We have explicitly solved the eigenvalue equation (eq. (3.4)). The results have confirmed that the $q$ - and $\Omega_{n}$ dependence of the eigenfunction $\phi\left(\boldsymbol{k}, \mathrm{i} \omega_{n}: \boldsymbol{q}, \mathrm{i} \Omega_{n}\right)$ is small in the region where the dominant contribution to the self-energy is yielded. In particular, it is confirmed that 
the value $1-\lambda\left(\boldsymbol{q}, \mathrm{i} \Omega_{n}\right)$ is found within the error of 10 20 par cent by this approximation. Thus, the second approximation only slightly underestimates the eigenvalue $\lambda\left(\boldsymbol{q}, \mathrm{i} \Omega_{n}\right)$ around $q=\Omega_{n}=0$, since the precise eigenfunction is determined so as to optimize the eigenvalue. Needless to say, the precise eigenvalue is obtained at $q=\Omega_{n}=0$. In other wards, the TDGL parameter (see eq. (3.10)) $b$ is overestimated by the second approximation whereas the mass term $t_{0}$ is obtained precisely. The approximation slightly underestimates the effects of the superconducting fluctuations. Since the approximation is not precise for large $q$ and $\Omega_{n}$, the unphysical contribution to the self-energy is caused by the region. Therefore, we eliminate the $q$ - and $\Omega_{n}$ independent term $g \phi\left(\boldsymbol{k}_{1}, \mathrm{i} \omega_{n}\right) \phi^{*}\left(\boldsymbol{k}_{2}, \mathrm{i} \omega_{m}\right)$ in the T-matrix.

By using the above two approximations, the T-matrix is expressed as follows,

$$
\begin{aligned}
& T\left(\boldsymbol{k}_{1}, \mathrm{i} \omega_{n}: \boldsymbol{k}_{2}, \mathrm{i} \omega_{m}: \boldsymbol{q}, \mathrm{i} \Omega_{n}\right) \\
& =\frac{g \lambda\left(\boldsymbol{q}, \mathrm{i} \Omega_{n}\right) \phi\left(\boldsymbol{k}_{1}, \mathrm{i} \omega_{n}\right) \phi^{*}\left(\boldsymbol{k}_{2}, \mathrm{i} \omega_{m}\right)}{1-\lambda\left(\boldsymbol{q}, \mathrm{i} \Omega_{n}\right)}
\end{aligned}
$$

where

$$
\begin{array}{r}
\lambda\left(\boldsymbol{q}, \mathrm{i} \Omega_{n}\right)=-T \sum_{\boldsymbol{k}, \mathrm{i} \omega_{n}} \sum_{\boldsymbol{p}, \mathrm{i} \omega_{m}} \phi^{*}\left(\boldsymbol{k}, \mathrm{i} \omega_{n}\right) V_{\mathrm{a}}\left(\boldsymbol{k}-\boldsymbol{p}, \mathrm{i} \omega_{n}-\mathrm{i} \omega_{m}\right) \\
G\left(\boldsymbol{p}, \mathrm{i} \omega_{m}\right) G\left(\boldsymbol{q}-\boldsymbol{p}, \mathrm{i} \Omega_{n}-\mathrm{i} \omega_{m}\right) \phi\left(\boldsymbol{p}, \mathrm{i} \omega_{m}\right) .(3.6)
\end{array}
$$

Here, the coupling constant $g$ is defined as,

$$
\begin{aligned}
g & =\sum_{\boldsymbol{k}_{1}, \mathrm{i} \omega_{n}} \sum_{\boldsymbol{k}_{2}, \mathrm{i} \omega_{m}} \phi^{*}\left(\boldsymbol{k}_{1}, \mathrm{i} \omega_{n}\right) \\
& \times V_{\mathrm{a}}\left(\boldsymbol{k}_{1}-\boldsymbol{k}_{2}, \mathrm{i} \omega_{n}-\mathrm{i} \omega_{m}\right) \phi\left(\boldsymbol{k}_{2}, \mathrm{i} \omega_{m}\right),
\end{aligned}
$$

and the wave function is normalized as,

$$
\sum_{\boldsymbol{k}, \mathrm{i} \omega_{n}}\left|\phi\left(\boldsymbol{k}, \mathrm{i} \omega_{n}\right)\right|^{2}=1 .
$$

By taking out the freedom of the superconducting fluctuations, we define the pair susceptibility as $t\left(\boldsymbol{q}, i \Omega_{n}\right)=$ $g /\left(1-\lambda\left(\boldsymbol{q}, \mathrm{i} \Omega_{n}\right)\right)$ which we have termed the T-matrix in the previous papers.19, 34, 33, 32, 31

The self-energy due to the superconducting fluctuations is given by the T-matrix approximation (Fig. $5(\mathrm{~b}))$.

$$
\begin{aligned}
\Sigma_{\mathrm{S}}\left(\boldsymbol{k}, \mathrm{i} \omega_{n}\right)= & T \sum_{\boldsymbol{q}, \mathrm{i} \Omega_{n}} T\left(\boldsymbol{k}, \mathrm{i} \omega_{n}: \boldsymbol{k}, \mathrm{i} \omega_{n}: \boldsymbol{q}, \mathrm{i} \Omega_{n}\right) \\
& \times G\left(\boldsymbol{q}-\boldsymbol{k}, \mathrm{i} \Omega_{n}-\mathrm{i} \omega_{n}\right) .
\end{aligned}
$$

In this section, we use the Green function determined by the FLEX approximation $G_{\mathrm{F}}\left(\boldsymbol{k}, \mathrm{i} \omega_{n}\right)=\left(\mathrm{i} \omega_{n}-\varepsilon_{\boldsymbol{k}}-\right.$ $\left.\Sigma_{\mathrm{F}}\left(\boldsymbol{k}, \mathrm{i} \omega_{n}\right)\right)^{-1}$ in calculating eqs. (3.5)-(3.9). That is to say, we calculate the lowest order correction due to the superconducting fluctuations on the FLEX approximation. We call the calculation FLEX+T-matrix approximation. The self-energy is obtained by the summation, $\Sigma\left(\boldsymbol{k}, \mathrm{i} \omega_{n}\right)=\Sigma_{\mathrm{F}}\left(\boldsymbol{k}, \mathrm{i} \omega_{n}\right)+\Sigma_{\mathrm{S}}\left(\boldsymbol{k}, \mathrm{i} \omega_{n}\right)$. We show the results of the FLEX+T-matrix approximation in the following subsections. Since the calculation is carried out with the fixed chemical potential $\mu$, the doping concentration $\delta$ decreases with decreasing the temperature $T$. However, the difference is smaller than 0.01 and has no significant effect.

In $\S 4$, we carry out the self-consistent calculation in which the fully dressed Green function $G\left(\boldsymbol{k}, \mathrm{i} \omega_{n}\right)=$ $\left(\mathrm{i} \omega_{n}-\varepsilon_{\boldsymbol{k}}-\Sigma_{\mathrm{F}}\left(\boldsymbol{k}, \mathrm{i} \omega_{n}\right)-\Sigma_{\mathrm{S}}\left(\boldsymbol{k}, \mathrm{i} \omega_{n}\right)\right)^{-1}$ is used everywhere. As a result of the self-consistency, the effect of the superconducting fluctuations are reduced. However, the qualitatively similar conclusions are obtained.

\subsection{Pseudogap in the single particle properties}

In this subsection the pseudogap, which is the main subject of this paper, is derived. The calculated results well justify the pairing scenario based on the self-epergy correction due to the superconducting fluctuations. 19, 29) On the basis of the model with a $d$-wave attractive interaction, it has been shown that the anomalous properties of the self-energy give rise to the pseudogap.19,29) The characteristics of the self-energy is that the real part has the positive slope and the imaginary part has the maximum absolute value at the Fermi level (see Fig. 8 in ref. 19). They are anomalous compared with the typical behaviors in the Fermi liquid theory (see Fig. 2). The large imaginary part reduces the single particle spectral weight at the Fermi level and gives rise to pseudogap. The anomalous features seem to compete with the Fermi liquid behaviors obtained by the FLEX calculations. In particular, the negative slope of the real part which is related to the renormalization factor $Z_{k}^{-1}$ is generally increased by the strong electron correlation. However, the calculated results clarify the important mechanism of the pseudogap formation in the strongly correlated electron systems.

We show the obtained results for the analytically continuated self-energy $\Sigma^{\mathrm{R}}(\boldsymbol{k}, \omega)$ in Fig. 6. Here, the doping concentration corresponds to the under-doped cuprates. It should be noticed that the Fermi liquid behaviors are seen when we look with the large energy scale $\omega \sim 0.5$. However, the anomalous behaviors leading to the pseudogap are clearly seen in much smaller energy scale $\omega \sim 0.05$. The anomalous behaviors vanish around $(\pi / 2, \pi / 2)$ because of the $d_{x^{2}-y^{2}}$ wave symmetry of the fluctuating Cooper pairs.

It is the important point that the superconductivity and the pseudogap take place in the renormalized quasiparticles near the Fermi surface. In other words, the pseudogap occurs with much smaller energy scale than that of the electron systems. The above results clearly show the smaller energy scale and justify the calculation based on the effective model in which a $d$-wave attractive interaction acts on the renormalized quasi-particles.

The results for the spectral weight are shown in Fig. 7. The pseudogap clearly appears in the single particle spectral weight (Fig. 7(a)) and the DOS (Fig. 7(b)).

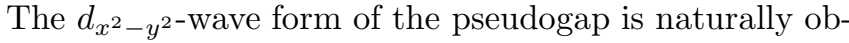
tained by the $d_{x^{2}-y^{2}}$-wave symmetry of the superconducting fluctuations (Fig. 7(a)). The inset of Fig. 7(b) shows the much smaller energy scale of the pseudogap compared with the band width. We wish to note again that the pseudogap is derived from the self-energy correction due to the superconducting fluctuations which are 
(a)

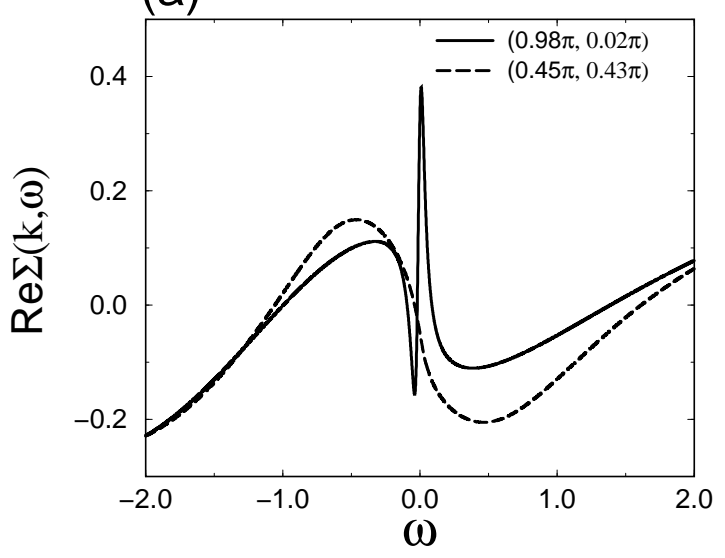

(b)

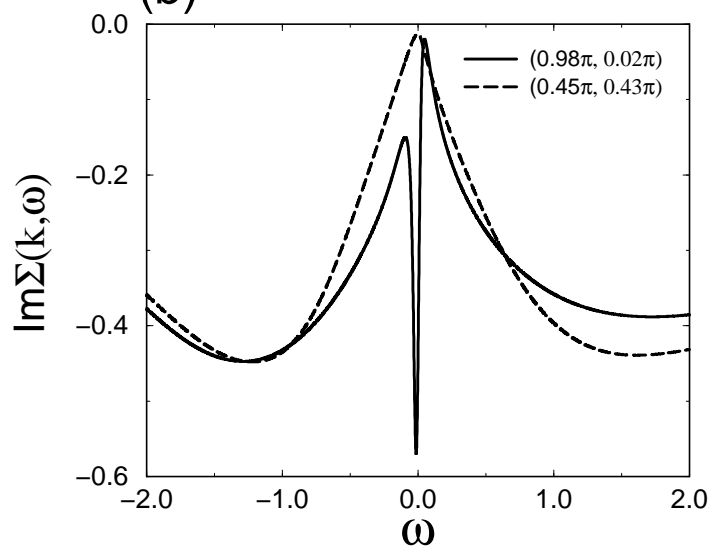

Fig. 6. The self-energy obtained by the FLEX+T-matrix approximation. (a)The real part. (b) The imaginary part. Here, $U=1.6, \delta=0.095$ and $T=0.010$. The solid line and the longdashed line correspond to $\left(\frac{63}{64} \pi, \frac{1}{64} \pi\right)$ (hot spot) and $\left(\frac{29}{64} \pi, \frac{27}{64} \pi\right)$ (cold spot), respectively.

enhanced by the strong couplino superconductivity and the quasi-two dimensionality. 19, 29, 31) The $\omega$-dependence of the wave function furthermore advances the pseudogap formation. Thus, the resonance scattering scenario is justified by the microscopic calculation based on the Hubbard model. It is microscopically confirmed that the superconducting fluctuations are sufficiently strong to give rise to the pseudogap.

The energy scale of the obtained pseudogap is consistent with that of the superconducting gap. The experimental results show that the energy scale of the pseudogap is nearly the same as that of the superconducting gap 13, 14. The ratio $2 \Delta_{\mathrm{S}} / T_{\mathrm{C}} \sim 12$ has been obtained by the FLEX approximation in the optimally-doped region 14) Here, $\Delta_{\mathrm{s}}$ is the maximum value of the gap function in the ordered state. The larger ratio is expected in the under-doped region. Our results for the under-doped cuprates show the ratio $2 \Delta_{\mathrm{pg}} / T_{\mathrm{c}} \sim 20$ (Fig. $7(\mathrm{a})$ ). Here $\Delta_{\text {pg }}$ is the energy scale of the pseudogap. Thus, the pseudogap obtained by our calculation seems to have the rel- evant energy scale compared with the superconducting gap.

(a)

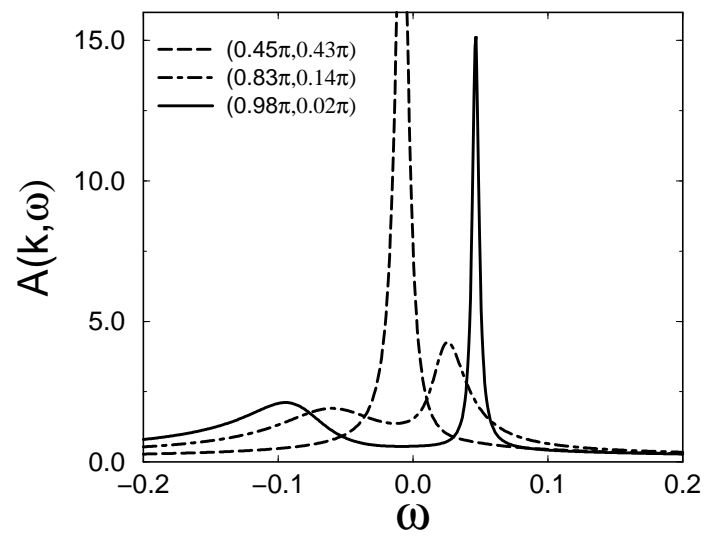

(b)

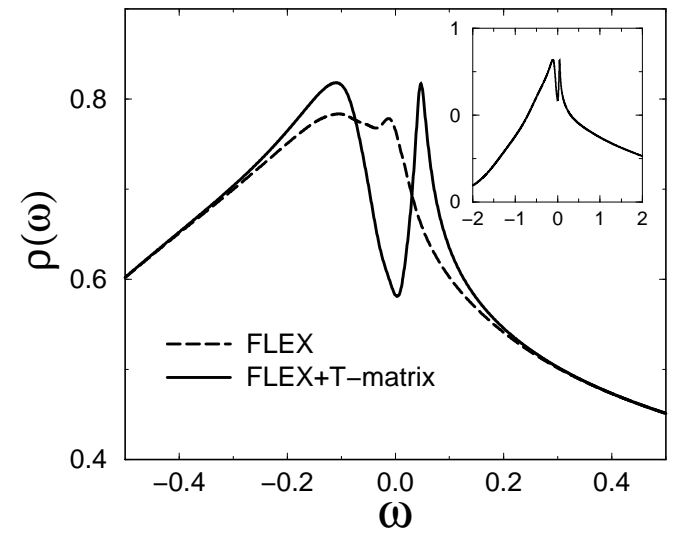

Fig. 7. (a)The single particle spectral weight obtained by the FLEX+T-matrix approximation. The solid and long-dashed lines correspond to those in Fig. 6, respectively. The dashdotted line corresponds to $\left(\frac{53}{64} \pi, \frac{9}{64} \pi\right)$. (b) The DOS obtained by the FLEX (long-dashed line) and the FLEX+T-matrix (solid line) approximations. The inset is the same result shown in the large energy scale. The other parameters are the same as those in Fig. 6.

We show the detailed results for the DOS in Fig. 8. We choose the temperature $T=1.25 T_{\mathrm{c}}$ in all figures of Fig. 8 in order to fix the distance to the critical point. The doping dependence is shown in Fig. 8(a). It is shown that the pseudogap becomes weak with increasing the hole-doping. The gap structure is filled up and the DOS near the Fermi level increases in the optimally-doped region. The effects of the superconducting fluctuations almost disappear in the over-doped region $\delta>0.2$. This is because both the critical temperature and the renormalization of the quasi-particles are reduced by the holedoping. Since the ratio $T_{\mathrm{c}} / \varepsilon_{\mathrm{F}}$ decreases, the superconducting coupling also decreases. Therefore, the effects of the superconducting fluctuations are reduced. Thus, the 
above calculation well explains the doping dependence of the pseudogap phenomena.

(a)

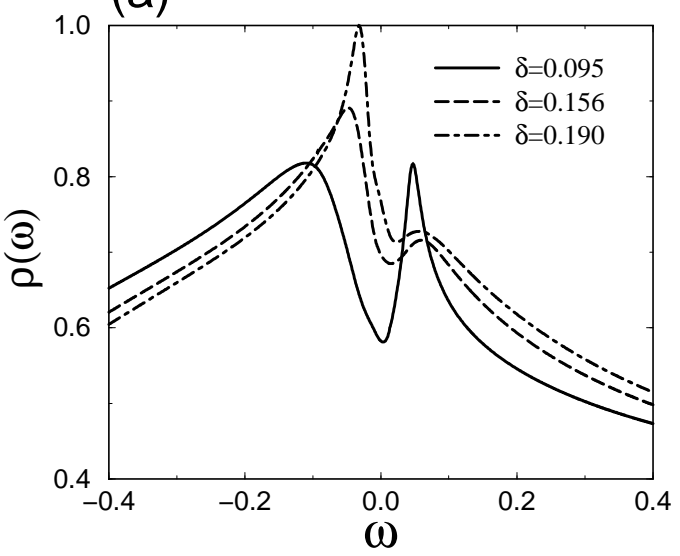

(b)

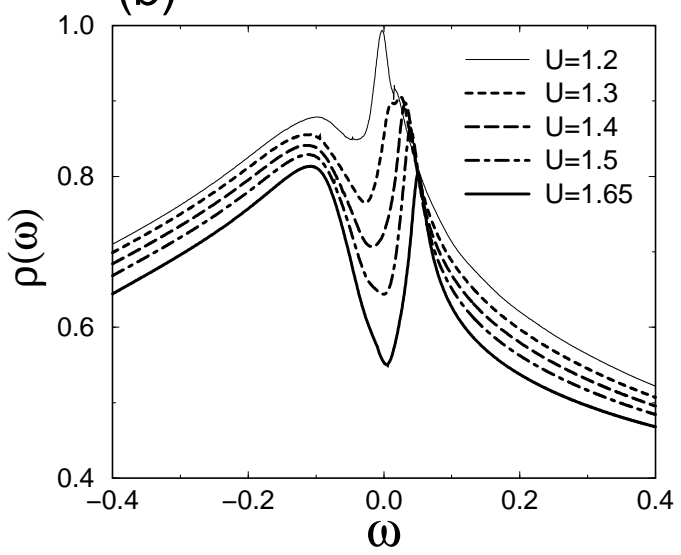

Fig. 8. (a) The doping dependence of the DOS obtained by the FLEX+T-matrix approximation. The solid, long-dashed and dash-dotted lines correspond to $\delta=0.095,0.156$ and 0.190 , respectively. (b) The density of state for the various strength of $U$. The thin solid, dashed, long-dashed, dash-dotted and thick solid lines correspond to $U=1.2,1.3,1.4,1.5$ and 1.65 , respectively. In all figures, the temperature is chosen as $T=1.25 T_{\mathrm{c}}$.

Here, the importance of the strong correlation is shown in Fig. 8(b). It is shown that the pseudogap becomes remarkable by increasing $U$. The reason is the same as that in the explanation of the doping dependence (Fig. 8(a)). Thus, the strong superconducting fluctuations and the resultant pseudogap are the characteristics of the strongly correlated electron systems.

\subsection{Superconducting fluctuations}

In this subsection, we discuss the character of the superconducting fluctuations in order to emphasize the importance of the strong coupling superconductivity.

In our pervious papers 19,34 the TDGL expansion is used for the pair susceptibility in the following way.

$$
t(\boldsymbol{q}, \omega)=\frac{g}{t_{0}+b \boldsymbol{q}^{2}-\left(a_{1}+\mathrm{i} a_{2}\right) \Omega},
$$

where the factors arising from the wave function $\phi\left(\boldsymbol{k}, \mathrm{i} \omega_{n}\right)$ are neglected. In the notation of this paper, the TDGL expansion corresponds to the expansion for the eigenvalue function $\lambda(\boldsymbol{q}, \Omega)$ as $1-\lambda(\boldsymbol{q}, \Omega)=$ $t_{0}+b \boldsymbol{q}^{2}-\left(a_{1}+\mathrm{i} a_{2}\right) \Omega$.

The TDGL expansion parameters generally describe the character of the superconducting fluctuations. The detailed properties of the TDGL parameters are discussed in ref. 19. Here, we discuss the TDGL parameters on the basis of the calculated results in this paper.

The parameter $t_{0}=1-\lambda(\mathbf{o}, 0)$ represents the distance to the phase transition, and is sufficiently small near $T_{\mathrm{c}}$. The parameter $a_{2}$ expresses the time scale of the fluctuations. The parameter $a_{1}$ is usually neglected within the weak coupling theory because it is higher order than $a_{2}$ with respect to the superconducting coupling $T_{\mathrm{c}} / \varepsilon_{\mathrm{F}}$. However, the parameter $\beta_{1}$ should not be neglected in the strong coupling case.19) The calculated results show that the sign of $a_{1}$ is negative in the under-doped region. The sign of $a_{1}$ which is fixed by the particle-hole asymmetry determines the sign of the Hall copductivity arising from the superconducting fluctuations.46,77) The Hall anomaly 46 ) has been explained by assuming the existence of the electron-like pre-formed pairs.28) However, it has been pointed out that the explanation is not necessarily justified within the theory of the strong coupling superconductivity.19)

The parameter $b$ represents the dispersion relation of the fluctuations and is related to the superconducting coherence length $\xi_{0}$ as $b \propto \xi_{0}^{2}$. The parameter $b$ is an important factor determining the strength of the fluctuations. The small $b$ generally means the strong superconducting fluctuations. Therefore, we show the results of the TDGL parameter $b$ in Fig. 9.

The TDGL parameter $b$ is expressed by the Fermi liquid description within the weak coupling theory as $b=g_{\mathrm{d}} \tilde{\rho}_{\mathrm{d}}(0) \frac{7 \zeta(3)}{32 \pi^{2}} \frac{\bar{v}_{\mathrm{F}}^{2}}{T^{2}}$. Here, $g_{\mathrm{d}}$ is the $d$-wave component of the residual interaction $Z_{\boldsymbol{k}} V_{\mathrm{a}}\left(\boldsymbol{k}-\boldsymbol{k}^{\prime}\right) Z_{\boldsymbol{k}^{\prime}}$, namely $g_{\mathrm{d}}=\sum_{\boldsymbol{k}, \boldsymbol{k}^{\prime}} \phi_{\mathrm{d}}(\boldsymbol{k}) Z_{\boldsymbol{k}} V_{\mathrm{a}}\left(\boldsymbol{k}-\boldsymbol{k}^{\prime}\right) Z_{\boldsymbol{k}^{\prime}} \phi_{\mathrm{d}}^{*}\left(\boldsymbol{k}^{\prime}\right)$. The effective density of state of the quasi-particles $\tilde{\rho}_{\mathrm{d}}(\varepsilon)$ is defined as $\tilde{\rho}_{\mathrm{d}}(\varepsilon)=\sum_{\boldsymbol{k}} \delta\left(\varepsilon-E_{\boldsymbol{k}}\right)\left|\phi_{\mathrm{d}}(\boldsymbol{k})\right|^{2}$, which is enhanced by the renormalization. Here, $E_{\boldsymbol{k}}$ is the quasiparticle's energy $E_{\boldsymbol{k}}=Z_{\boldsymbol{k}}\left(\varepsilon_{\boldsymbol{k}}+\operatorname{Re} \Sigma^{\mathrm{R}}(\boldsymbol{k}, 0)\right)$. The $\bar{v}_{\mathrm{F}}$ is the effective Fermi velocity for the $d$-wave symmetry. This is defined as $\bar{v}_{\mathrm{F}}^{2}=\int_{\mathrm{F}} \tilde{\rho}_{k_{\mathrm{F}}} \tilde{v}\left(k_{\mathrm{F}}\right)^{2} \mathrm{~d} k_{\mathrm{F}} / \tilde{\rho}_{\mathrm{d}}(0)$, where the integration is carried out on the Fermi surface and $\tilde{\rho}_{k_{\mathrm{F}}}=\left|\phi_{\mathrm{d}}\left(k_{\mathrm{F}}\right)\right|^{2} / \tilde{v}\left(k_{\mathrm{F}}\right)$. The velocity $\tilde{v}\left(k_{\mathrm{F}}\right)$ is the absolute value of the quasi-particle's velocity $\tilde{\boldsymbol{v}}_{\boldsymbol{k}}=\mathrm{d} E_{\boldsymbol{k}} / \mathrm{d} \boldsymbol{k}$ on the Fermi surface. It should be noticed that the quasiparticle's velocity is reduced by the renormalization, especially around $(\pi, 0)$. The renormalization is caused by the mass renormalization $Z_{\boldsymbol{k}}$ and the transformation of the Fermi surface. The bare velocity $\boldsymbol{v}_{\boldsymbol{k}}=\mathrm{d} \varepsilon_{\boldsymbol{k}} / \mathrm{d} \boldsymbol{k}$ is small at the 'hot spot' on the transformed Fermi surface. Since the effective Fermi velocity $\bar{v}_{\mathrm{F}}$ is mainly determined by the region around $(\pi, 0)$, the velocity $\bar{v}_{\mathrm{F}}$ is remarkably 
(a)

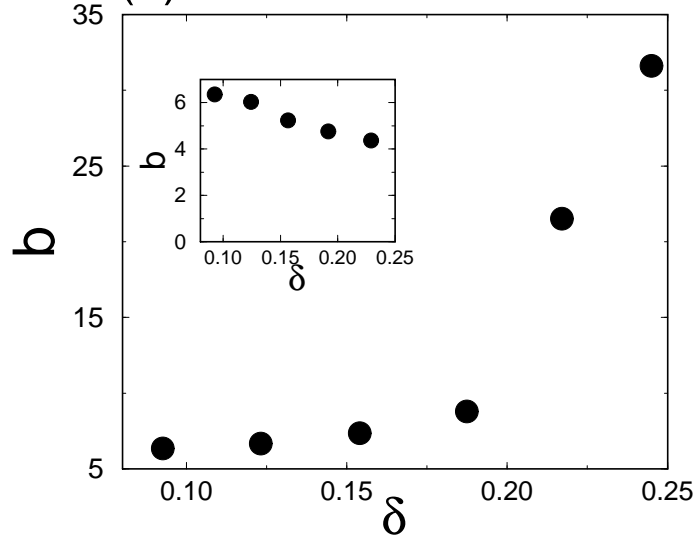

(b)

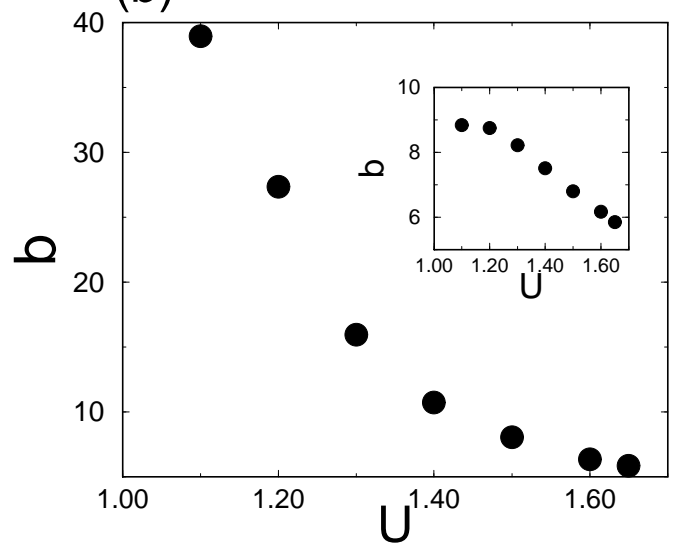

Fig. 9. (a) The doping dependence of the TDGL parameter $b$ at the critical point $T=T_{\mathrm{c}}$. Here, the coupling constant is fixed to $U=1.6$. The inset shows the same quantity at the fixed temperature $T=0.0080$ (b) The TDGL parameter $b$ for the various strength of $U$ at $T=T_{\mathrm{c}}$. Here the doping concentration is fixed to $\delta=0.09$. The inset shows the same quantity at the fixed temperature $T=0.0082$

reduced by the electron correlation.

It should be noticed that the TDGL parameter $b$ is proportional to $\bar{v}_{\mathrm{F}}^{2} / T^{2}$, which is proportional to the inverse square of the superconducting coupling $T_{\mathrm{c}} / \varepsilon_{\mathrm{F}}$. In this sense, the effective Fermi energy should be defined as $\varepsilon_{\mathrm{F}} \propto \bar{v}_{\mathrm{F}}$. That is to say, the TDGL parameter $b$ decreases with increasing the superconducting coupling. We can see in Fig. 9 that the TDGL parameter $b$ decreases with under-doping and/or with increasing $U$. It is because the critical temperature $T_{\mathrm{c}}$ increases and the renormalization for the effective Fermi velocity $\bar{v}_{\mathrm{F}}$ becomes remarkable. These effects reduce the TDGL parameter $b$ in spite of the increasing coupling constant $g_{\mathrm{d}} \tilde{\rho}_{\mathrm{d}}(0)$. The effects of the quasi-particle's renormalization is confirmed by showing the results at the fixed temperature. (see the inset in Fig. 9(b)) Although the coupling constant $g_{\mathrm{d}} \tilde{\rho}_{\mathrm{d}}(0)$ increases and the temperature $T$ is fixed, the parameter $b$ decreases with increasing $U$. This is because the effective Fermi velocity $\bar{v}_{\text {F }}$ is reduced.

Thus, the superconductivity becomes strong coupling one when the electron correlation is strong. The above results microscopically justify our scenario about the doping dependence of the pseudogap phenomena. The superconducting fluctuations become strong, and the pseudogap phenomena take place in the wide temperature region with decreasing the doping concentration $\delta$. These doping dependence is consistent with the experimental results. Moreover, the above doping dependence of the parameter $b$ is consistent with the magnetic field dependence of the pseudogap phenomena. $34.49,50$.51.52)

\subsection{Magnetic properties}

In this subsection, we show the results for the magnetic properties which are measured by NMR, neutron scattering and so on. The anti-ferromagnetic spin fluctuations are one of the important characters of High$T_{\mathrm{c}}$ cuprates. The development of the spin fluctuations are well described by the FLEX approximation. One the other hand, the suppression of the low frequency spin fluctuations have been pointed out by NMR mea-

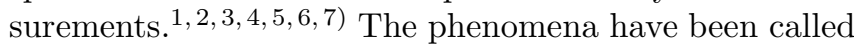
'spin gap' at the initial stage. At present, it is known that not only the spin channel but also the single particle properties show the gap-like features 13, 14 Therefore, the 'spin gap' is considered to be a result of the gap formation in the single particle properties, namely 'pseudogap'. In this subsection, the pseudogap phenomena in the magnetic properties are explained by considering the effects of the superconducting fluctuations on the single particle properties.

In the FLEX+T-matrix approximation, the spin susceptibility $\chi_{\mathrm{s}}(\boldsymbol{q}, \Omega)$ is obtained by extending the FLEX approximation,

$$
\begin{aligned}
\chi_{\mathrm{s}}^{\mathrm{R}}(\boldsymbol{q}, \Omega) & =\frac{\chi_{0}^{\mathrm{R}}(\boldsymbol{q}, \Omega)}{1-U \chi_{0}^{\mathrm{R}}(\boldsymbol{q}, \Omega)}, \\
\chi_{0}\left(\boldsymbol{q}, \mathrm{i} \Omega_{n}\right) & =-T \sum_{\boldsymbol{k}, \mathrm{i} \omega_{n}} G\left(\boldsymbol{k}, \mathrm{i} \omega_{n}\right) G\left(\boldsymbol{k}+\boldsymbol{q}, \mathrm{i} \omega_{n}+\mathrm{i} \Omega_{n}\right),
\end{aligned}
$$

where the dressed Green function $G\left(\boldsymbol{k}, \mathrm{i} \omega_{n}\right)=\left(\mathrm{i} \omega_{n}\right.$ $\left.\varepsilon_{\boldsymbol{k}}-\Sigma_{\mathrm{F}}\left(\boldsymbol{k}, \mathrm{i} \omega_{n}\right)-\Sigma_{\mathrm{S}}\left(\boldsymbol{k}, \mathrm{i} \omega_{n}\right)\right)^{-1}$ is used. The effects of the superconducting fluctuations are included in the selfenergy, $\Sigma_{\mathrm{S}}\left(\boldsymbol{k}, \mathrm{i} \omega_{n}\right)$.

The NMR spin-lattice relaxation rate $1 / T_{1}$ and spinecho decay rate $1 / T_{2 \mathrm{G}}$ are obtained by the following formula.

$$
\begin{aligned}
& 1 / T_{1} T=\sum_{\boldsymbol{q}} F_{\perp}(\boldsymbol{q})\left[\left.\frac{1}{\omega} \operatorname{Im} \chi_{\mathrm{s}}^{\mathrm{R}}(\boldsymbol{q}, \omega)\right|_{\omega \rightarrow 0}\right], \\
& 1 / T_{2 G}^{2}=\sum_{\boldsymbol{q}}\left[F_{\|}(\boldsymbol{q}) \operatorname{Re} \chi_{\mathrm{s}}^{\mathrm{R}}(\boldsymbol{q}, 0)\right]^{2}-\left[\sum_{\boldsymbol{q}} F_{\|}(\boldsymbol{q}) \operatorname{Re} \chi_{\mathrm{s}}^{\mathrm{R}}(\boldsymbol{q}, 0)\right]^{2} .
\end{aligned}
$$

Here, $F_{\perp}(\boldsymbol{q})=\frac{1}{2}\left[\left\{A_{1}+2 B\left(\cos q_{x}+\cos q_{y}\right)\right\}^{2}+\left\{A_{2}+\right.\right.$ $\left.\left.2 B\left(\cos q_{x}+\cos q_{y}\right)\right\}^{2}\right]$ and $F_{\|}(\boldsymbol{q})=\left\{A_{2}+2 B\left(\cos q_{x}+\right.\right.$ $\left.\left.\cos q_{y}\right)\right\}^{2}$. The hyperfine coupling constants $A_{1}, A_{2}$ and $B$ are evaluated as $A_{1}=0.84 B$ and $A_{2}=-4 B .88$ 
The calculated results for the NMR $1 / T_{1} T, 1 / T_{2 \mathrm{G}}$ and the static spin susceptibility are shown in Fig. 10. The results show the pseudogap in the NMR $1 / T_{1} T$ (Fig. 10(a)). In the FLEX calculation, the NMR $1 / T_{1} T$ increases with decreasing the temperature (see the inset in Fig. 10(a)). This corresponds to the Curie-Weiss low of the spin fluctuations. In the FLEX+T-matrix calculation, the NMR $1 / T_{1} T$ increases with decreasing the temperature from high temperature, shows its peak at $T^{*}$ and decreases with decreasing the temperature. This decrease above the critical point $T=T_{0}$ is the vell-known pseudogap in NMR measurements. nomenon is caused by the superconducting fluctuations. Since the DOS is reduced by the superconducting fluctuations, the low frequency spin fluctuations are suppressed. Because the NMR $1 / T_{1} T$ measures the low frequency component of the spin fluctuations, $1 / T_{1} T$ decreases with approaching the critical point. Thus, the pseudogap observed in NMR $1 / T_{1} T$ takes place through the pseudogap in the single particle properties.

The NMR $1 / T_{2 \mathrm{G}}$ also shows the pseudogap (Fig. 10(b)) with the same onset temperature $T^{*}$ as that in $1 / T_{1} T$. The NMR $1 / T_{2 \mathrm{G}}$ decreases with approaching the critical point. This is also an effect of the superconducting fluctuations. However, the pseudogap in the NMR $1 / T_{2 \mathrm{G}}$ is weak compared with that in the NMR $1 / T_{1} T$. This is because the NMR $1 / T_{2 \mathrm{G}}$ measures the static spin susceptibility which reflects the total weight of the spin fluctuations.33. 34 It should be noticed that the pseudogap suppresses only the low frequency component of the spin fluctuations. This is a natural result because the superconductivity has a smaller energy scale than that of the spin fluctuations. Thus, it is no wonder that the scaling relation of the spin fluctuations is violated in the pseudogap state. for the NMR give the qualitatively consistent explana-

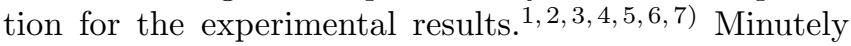
speaking, the different behaviors of $1 / T_{2}$ have been reported for different High- $T_{\mathrm{c}}$ compounds. 34 , There is an idea that attributes the difference to the effects of the interlayer coupling.(6) The results of the single layer compqunds show the decrease of $1 / T_{2 G}$ in the pseudogap state. Anyway, the relatively weak effect of the pseudogap on $1 / T_{2 G}$ than on $1 / T_{1} T$ is observed in common, which is consistent with our results.

The similar features of the NMR $1 / T_{1} T$ and $1 / T_{2 \mathrm{G}}$ have been observed in the superconducting state.t. In particular, the $1 / T_{2 \mathrm{G}}$ remains even in the low temperature, although the $1 / T_{1} T$ rapidly decreases. These features are the characteristics of the $d$-wave superconductivity.33.79) Therefore, the above results for the pseudogap state are natural because the pseudogap is a precursor of the $d$-wave superconductivity.

While the many quantities shgw the pseudogap with the same onset temperature $T^{*}, 10$ ) the uniform spin susceptibility $\chi_{\mathrm{s}}^{\mathrm{R}}(\mathbf{o}, 0)$ decreases from the much higher temperature than $T^{*}$. However, the decrease of the uniform susceptibility becomes more rapid near $T^{*}$ 目 $\mathrm{We}$ consider that the rapid decrease is caused by the superconducting fluctuations. The calculated results con-
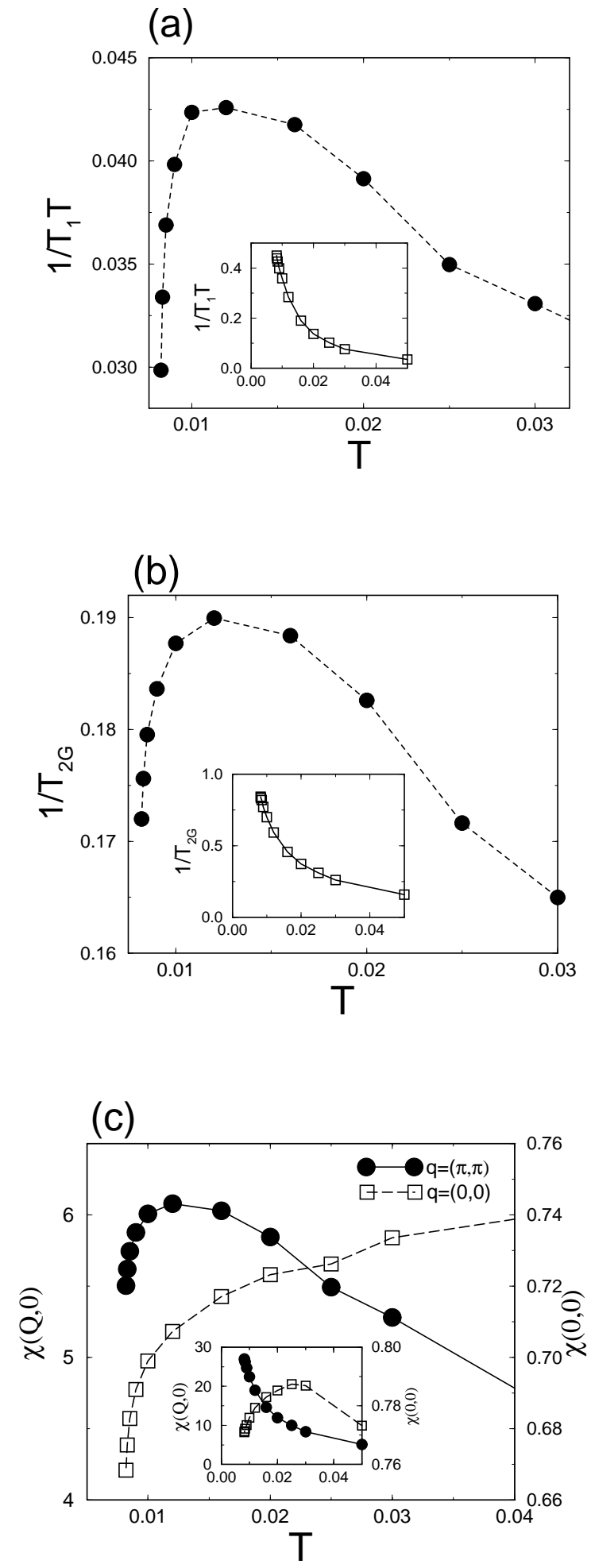

Fig. 10. The temperature dependence of (a) the NMR $1 / T_{1} T$ and (b) the NMR $1 / T_{2 \mathrm{G}}$ calculated by the FLEX+T-matrix approximation. (c) The temperature dependence of static spin susceptibility $\chi_{\mathrm{s}}^{\mathrm{R}}(\boldsymbol{q}, 0)$ at $\boldsymbol{q}=(0,0)$ (Open squares) and at $\boldsymbol{q}=(\pi, \pi)$ (Closed circles). Here, the doping concentration is fixed to the under-doped region $\delta=0.093 \sim 0.103$. The inset in (a), (b) and (c) shows the same quantities calculated by the FLEX approximation. 
firm the consideration. We show the results for the uniform susceptibility $\chi_{\mathrm{s}}^{\mathrm{R}}(\mathbf{o}, 0)$ and the staggard susceptibility $\chi_{\mathrm{s}}^{\mathrm{R}}(\boldsymbol{Q}, 0)$ in Fig. $10(\mathrm{c})$. The staggard susceptibility shows the pseudogap at the pseudogap onset temperature $T^{*}$ observed in $1 / T_{1} T$. On the other hand, the uniform susceptibility $\chi_{\mathrm{S}}^{\mathrm{R}}(\mathbf{o}, 0)$ decreases with decreasing the temperature from much higher temperature. The decrease becomes rapid near $T^{*}$. These results well explain results of the NMR measurements.5

The slight decrease of the uniform susceptibility is shown even in the FLEX approximations. (see the inset in Fig. 10(c).) Therefore, the decrease of the uniform susceptibility is not necessarily attributed to the superconducting fluctuations. However, the superconducting fluctuations significantly affect the uniform susceptibility and remarkably reduce the quantity near the critical point.

The frequency dependence of the spin susceptibility well describes the character of the pseudogap in the magnetic properties. The results for the dynamical spin susceptibility $\chi_{\mathrm{s}}^{\mathrm{R}}(\boldsymbol{q}, \Omega)$ at $\boldsymbol{q}=\boldsymbol{Q}$ is shown in Fig. 11 . The real part is suppressed at the low frequency in the pseudogap state $(T=0.0082$ in Fig. $11(\mathrm{a}))$. Thus, the magnetic order is suppressed by the superconducting fluctuations.

The imaginary part has been measured by the inelastic neutron scattering and shows the pseudogap 8) The calculated results show that the imaginary part is remarkably suppressed at the low frequency in the pseudogap state (Fig. 11(b)). This is the pseudogap phenomenon observed by the neutron scattering measurements. On the other hand, the spin fluctuations develop in higher frequency region. In other words, the pseudogap transfers the spectral weight of the spin fluctuations from the low frequency region to the high frequency region. Therefore, the total weight is not so reduced by the pseudogap. These features are consistent with the experimental results 8 ) and with the above explanation for the NMR $1 / T_{1} T$ and $1 / T_{2}$. It is notable that the pairing interaction arising from the spin fluctuations originates in the relatively wide frequency region. Therefore, the $d$-wave pairing interaction is not so reduced by the pseudogap. The interplay between the superconducting fluctuations and the spin fluctuations will be discussed in $\S 4.1$.

In the remaining part of this subsection, we discuss the commensurate and incommensurate structure of the spin fluctuations. The incommensurability in the Y-based compounds $\mathrm{YBa}_{2} \mathrm{Cu}_{3} \mathrm{O}_{6+\delta}$ has been pointed out by the inelastic neutron scattering measurements, and has been discussed in connection with the stripe phase 81) The stripe phase in the La-based compounds $\mathrm{La}_{2-x} \mathrm{Sr}_{x} \mathrm{CuO}_{4}$ has been measured by the elastic nejtron scattering and has been actively discussed lately. $82,83,84,85,86,87,88,89$

It should be noticed that both commensurate and incommensurate structures are obtained within the FLEX approximation (Fig. 12(a) and 12(c)). The incommensurability $\delta_{\text {inc }}$ is defined by the peak position of the dynamical spin susceptibility $\boldsymbol{Q}_{\mathrm{p}}=\left(\pi \pm \delta_{\text {inc }}, \pi\right)$ and $\left(\pi, \pi \pm \delta_{\text {inc }}\right)$. Whether the spin fluctuations are commensurate or in-
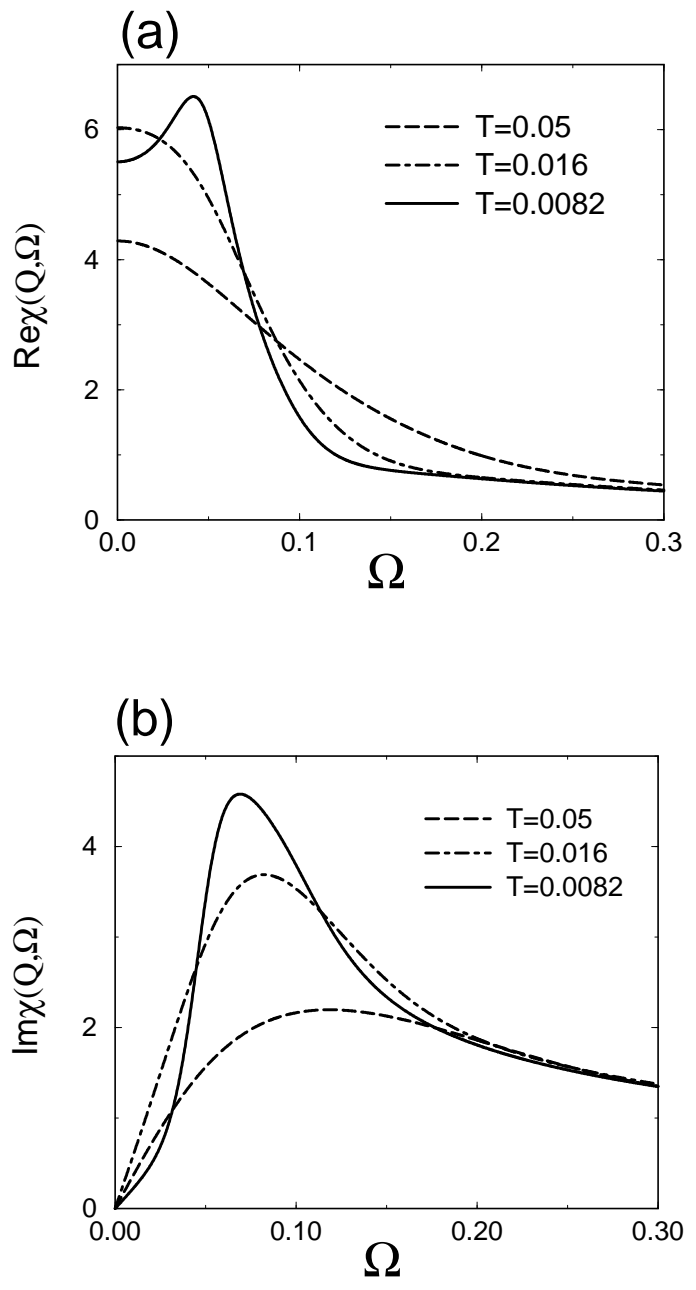

Fig. 11. The frequency dependence of the staggard spin susceptibility $\chi_{\mathrm{s}}^{\mathrm{R}}(\boldsymbol{Q}, \Omega)$ calculated by the FLEX+T-matrix approximation for under-doped cuprates. (a) The real part. (b) The imaginary part. The solid lines, the dash-dotted lines and the long-dashed lines correspond to $T=0.0082, T=0.016$ and $T=0.05$, respectively.

commensurate is determined by the chosen parameters, $t^{\prime}, \delta$ and $U$. One of the general results in our calculation is that the incommensurability $\delta_{\text {inc }}$ increases with the doping concentration $\delta$. These results are qualitatively consistent with the experimental results.90) The detailed agreement with the experimental results has been discussed by the FLEX calculation in the normal state.91)

The other general result is that the superconducting fluctuations enhance the incommensurability. Although the commensurate peak is obtained by the FLEX approximation in the under-doped region (Fig. 12(a)), it becomes incommensurate owing to the superconducting fluctuations (Fig. 12(b)). The incommensurability $\delta_{\text {inc }}$ increases in the FLEX+T-matrix approximation in the optimally-doped region (Fig. 12(d)). The above effect also originates in the pseudogap formation around $(\pi, 0)$. The results well explain the experimental results of the inelastic neutron scattering. The neutron scattering ex- 
(a) under-doped (FLEX)

$\operatorname{Im} \chi(q, \Omega)$

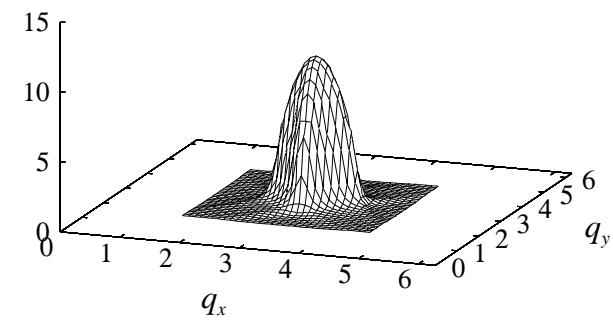

(b) under-doped (FLEX+T-matrix)

$\operatorname{Im} \chi(q, \Omega)$

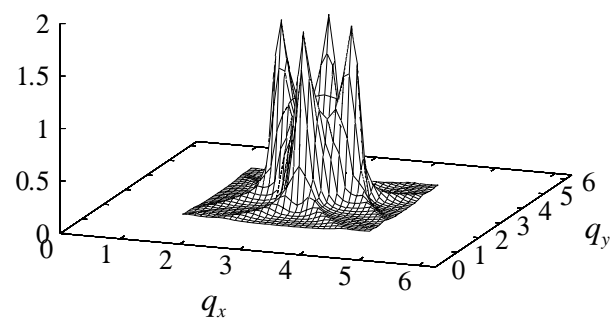

(c) optimally-doped (FLEX)

$\operatorname{Im} \chi(q, \Omega)$

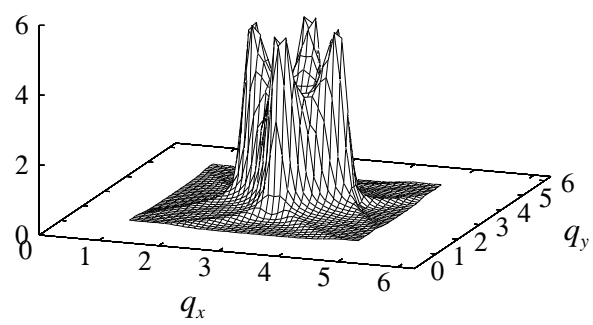

(d) optimally-doped (FLEX+T-matrix)

$\operatorname{Im} \chi(q, \Omega)$

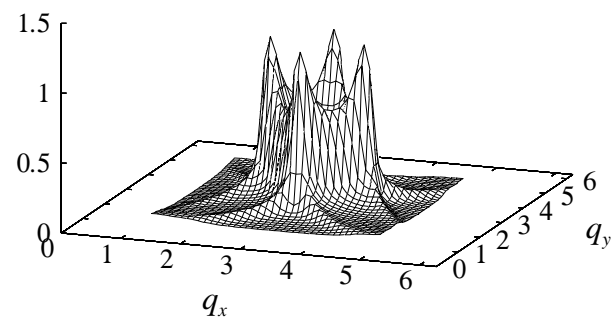

Fig. 12. The momentum dependence of the dynamical spin susceptibility $\operatorname{Im} \chi_{\mathrm{S}}^{\mathrm{R}}(\boldsymbol{q}, \Omega)$ at $\Omega=0.01$. The results for under-doped cuprates $(\delta=0.095$ and $T=0.010)$ (a) by the FLEX approximation and (b) by the FLEX+T-matrix approximation. The periments have shown that the spin fluctuations change from incommensurate to fommensurate with increasing the measured frequency.81) Although the measurements have been carried out in the superconducting state in many cases, the qualitatively similar behaviors as those in the pseudogap state are expected in the superconducting state. This is because the pseudogap and the

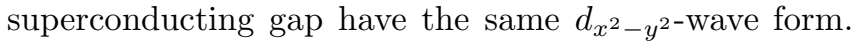
The effect of the pseudogap or the superconducting gap disappears when the measurement is done at the higher frequency than the energy scale of the gap. Thus, the incommensurate peak in the inelastic neutron scattering can be explained without any assumption of the charge and/or spin spatial modulation. The calculation including the superconducting order is desired for the detailed agreement with the experimental results.

\subsection{Electron-doped cuprates}

In this subsection, we apply the above calculation to the electron-doped cuprates. It is well known that not only the hole-doped cuprates but also the electron-doped cuprates such as $\mathrm{Nd}_{2-x} \mathrm{Ce}_{x} \mathrm{CuO}_{4-y}$ and $\mathrm{Pr}_{2-x} \mathrm{Ce}_{x} \mathrm{CuO}_{4-y}$ are the superconductors 92 ) The electron-hole symmetry is expected within the simple Hubbard model including only the nearest-neighbor hopping $t$. However, some different properties from the holedoped cuprates have been pointed out for the electrondoped cuprates. The anti-ferromagnetic ordered state is robust against the electron doping rather than the hole doping. The relatively low superconducting critical temperature $T_{\mathrm{c}}$ is observed in the narrow doping range.92)

It has been suggested for a long time that the electron-doped cuprates are an $s$-wave or the other node-less superconductor. Some experiments have supported the node-less superconductivity. The exponential dependence of the magnetic penetration depth has been reported.93) The absence of the zero bias conductance peak is also reported 94 , while it should exist in the $d$-wave superconductor.95) The possibility of some node-less superconductivity has been proposed theoretically. 9 ) However, some recent experimental results support the $d_{x^{2}-y^{2}}$-wave superconductivity also in the electron-doped cuprates The power law of the magneticpenetration depth 97 ) and the zero bias conductance peak 98 are shown. The $d_{x^{2}-y^{2}}$-wave form of the superconducting gap is directly measured by the ARPES. 2 ) Moreover, the phase sensitive evidence for the $d$-wave superconductivity is also reported by the SQUID microscope.100

We consider that the hole- and electron-doped cuprates should be understood comprehensively by the theory including their respective characters. The anomalous properties of the Hall coefficient are well explained for not only hole- but also electron-doped cuprates on the basis of the same formalism treating the spin fluctuations.72, 83 ) Therefore, it is natural to expect the same mechanism for the superconductivity arising from the spin fluctuations. Actually, the instability of the $d$-wave superconductivity mediated by the spin fluctuations has been reported theoretically. 72,101, , 102) 
We consider that the application to the electron-doped cuprates is an important test of the theories on High- $T_{\mathrm{c}}$ cuprates. Here, it is shown that our theory properly describes the important properties of the electron-doped cuprates. The electron-hole asymmetric properties are introduced by the next-nearest-neighbor hopping term $t^{\prime}$ in our model. The features of the electron-hole asymmetry are naturally explained by our calculations. Moreover, some theoretical expectations are proposed below. The experimental verification is desirable.

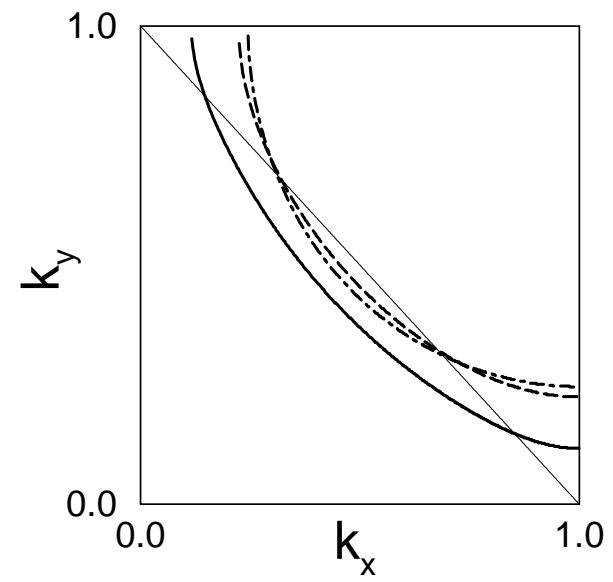

Fig. 13. The Fermi surface of the hole- and the electron-doped cuprates. The thick solid line shows the Fermi surface of the holedoped case $\delta=0.10$. The long-dashed and the dash-dotted lines show the Fermi surface of the electron-doped case $\delta=-0.10$. The long-dashed and the dash-dotted lines correspond to case $t^{\prime}=-0.25 t$ and $t^{\prime}=-0.35 t$, respectively. The thin solid line shows the magnetic Brillouin zone. It should be mentioned that this figure shows the non-interacting Fermi surface. Actually, the Fermi syrface is transformed by the anti-ferromagnetic spin fluctuations.56

The main difference between the electron- and holedoped cuprates results from the shape of the Fermi surface. The distance from the Fermi surface to the VanHove singularity is especially important. The Fermi surface of the electron-doped cuprates is obtained by lifting the chemical potential $\mu$. The typical Fermi surface is shown in Fig. 13. The Fermi level is lifted from the Van-Hove singularity $(\pi, 0)$ where the dispersion is flat. Therefore, the DOS is rather small in the electron-doped cuprates. The small DOS means that the electron correlation is effectively weak. On the other hand, the nesting around $(\pi / 2, \pi / 2)$ is enhanced and the tendency toward the anti-ferromagnetic order is robust. In the light of the $d-p$ model, the carrier is confined in the $\mathrm{Cu}$-site in the electron-doped cases, while it is mainly in the O-site in the hole-doped ones. This fact probably contributes to the robustness of the anti-ferromagnetic order. This difference may affect the parameter of the Hubbard model which is an effective model of the $d-p$ model in the metallic phase. However, the essential difference is included in the properties of the band structure since only the vicinity of the Fermi surface is important for the low energy physics.
Since we define the doping concentration as $\delta=1-n$, it is negative in the electron-doped case. We should note that the numerical calculation is not so correct for the electron-doped cases as for the hole-doped ones because of some reasons. The main reason is the finite size effects which are serious owing to the large velocity, the weak correlation and the low temperature. The finite size effects on the spin- and superconducting fluctuations are also serious by the same reasons. Therefore, we divide the first Brillouin zone into $128 \times 128$ lattice points in the calculation for the electron-doped cases.

For example, the difference from the hole-doped cuprates appears in the magnetic properties. We show the results of the FLEX approximation for the momentum dependence of the dynamical spin susceptibility in Figs. 14 (a) and (b). In these figures, we use the same parameters $t, t^{\prime}$ and $U$ as those of the hole-doped cases. A remarkable feature of the spin correlation is that the range of the spin fluctuations is narrow in the momentum space. The width of the range and the strength of the anti-ferromagnetic correlation are reduced by increasing the electron-doping.

It is notable that the FLEX calculation is difficult untill the electron-doping $|\delta| \sim 0.12$ because the antiferromagnetic correlation is too strong. This fact implies that the anti-ferromagnetic order is robust in the electron-doped cuprates than in the hole-doped ones. The narrow range of the spin fluctuations means that the mode coupling effects are weak in the electrondoped cuprates. This favors the anti-ferromagnetic order. The other factors probably contribute to the robustness of the anti-ferromagnetism. For example, the anti-ferromagnetic order is robust when the three dimensionality (or the interlayer coupling) is strong. Moreover, the frustration due to the carrier doping is weak when the carrier enters the $\mathrm{Cu}$-site.

Hereafter, we discuss the superconductivity and the pseudogap in the electron-doped cuprates. The superconductivity mediated by the spin fluctuations is also derived by using the FLEX approximation. The most

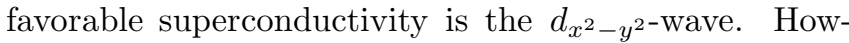
ever, the superconducting critical temperature is very low. The critical temperature $T_{\mathrm{c}}$ higher than 0.002 is not obtained in case of the parameter $t^{\prime}=0.25 t$. This is mainly because the pairing interaction mediated by the spin fluctuations is weak in the electron-doped case. The narrow range of the spin fluctuations in the momentum space weakens the pairing interaction. It should be noticed that the pairing interaction results from the spin fluctuations in the wide region around $\boldsymbol{Q}=(\pi, \pi)$. In addition, the small DOS reduces the critical temperature much more. Moreover, the Fermi surface in the electrondoped case is disadvantageous to the $d_{x^{2}-y^{2}}$-wave superconductivity, compared with the hole-doped case.

We can obtain a higher critical temperature $T_{\mathrm{c}}$ by choosing the hopping parameter $t^{\prime}$ so as to reproduce the Fermi surface of $\mathrm{Nd}_{2-x} \mathrm{Ce}_{x} \mathrm{CuO}_{4-y}$ with more accuracy. Here, we choose $t^{\prime}=0.35 t$ and $U=2.0$. The commensurate spin fluctuations over a wider range are obtained (Figs. 14(c) and (d)). The spin fluctuations give rise to 
(a) $\delta=-0.12$

$\operatorname{Im} \chi(q, \Omega)$

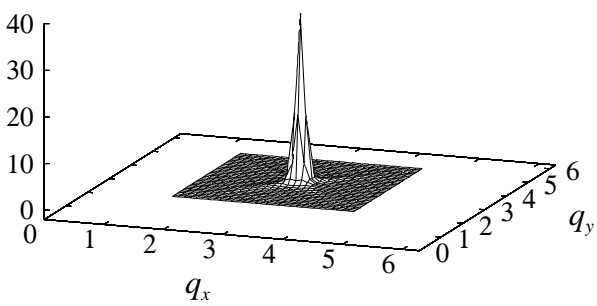

(b) $\delta=-0.15$

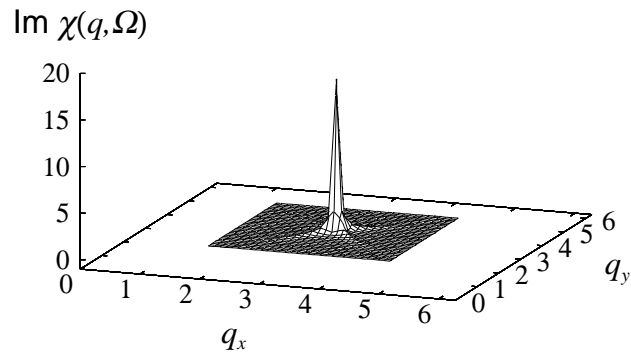

(c) $\delta=-0.10$

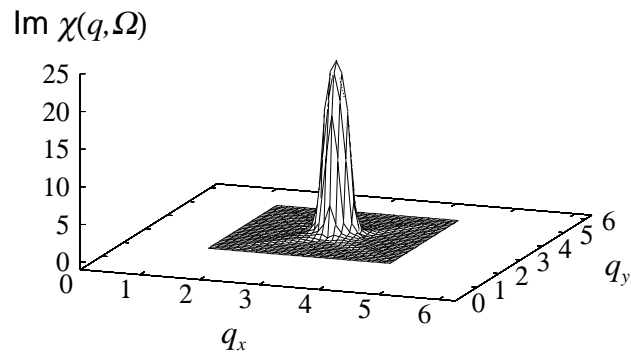

(d) $\delta=-0.13$

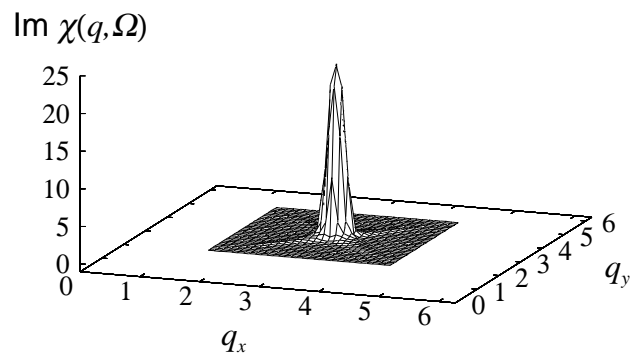

Fig. 14. The momentum dependence of the dynamical spin susceptibility $\operatorname{Im} \chi_{\mathrm{s}}^{\mathrm{R}}(\boldsymbol{q}, \Omega)$ at $\Omega=0.01$ calculated by the FLEX approximation. (a) $\delta=-0.123$ and (b) $\delta=-0.150$ at $t^{\prime}=-0.25 t$ and $T=0.01$. (c) $\delta=-0.104$ and (d) $\delta=-0.130$ at $t^{\prime}=-0.35 t$ the $d_{x^{2}-y^{2} \text {-wave superconductivity. However, the critical }}$ temperature is low and the doping range in which the superconductivity occurs is remarkably narrow compared with the hole-doped ones (see the inset of Fig. 15). The maximum value of the obtained critical temperature is $T_{\mathrm{c}}=0.0045$. The doping range is $\delta=-0.102 \sim-0.107$. The results for the superconductivity are qualitatively consistent with the experimental results.92

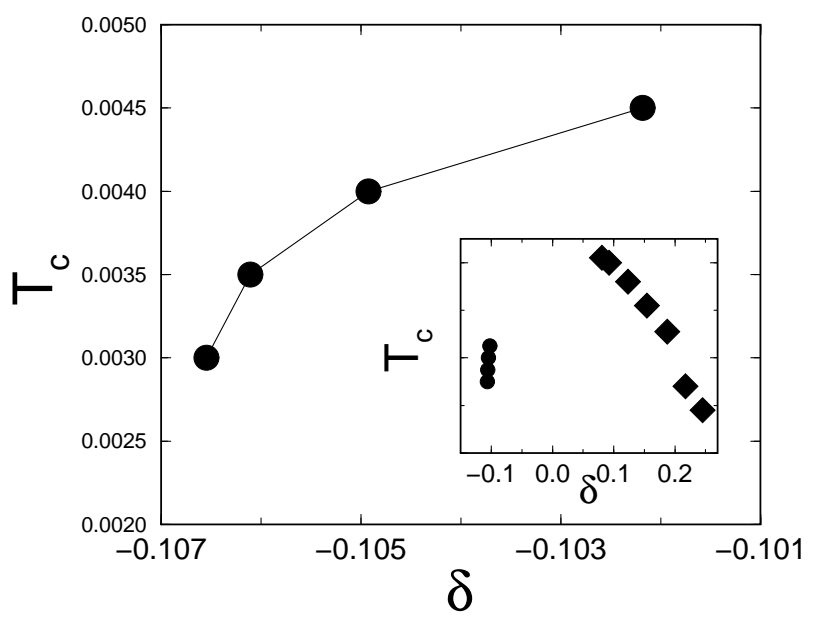

Fig. 15. The obtained critical temperature $T_{\mathrm{c}}$ in the electrondoped case. The inset shows the phase diagram including both hole- and electron-doped cases.

We show the momentum dependence of the wave function of the Cooper pairs $\phi\left(\boldsymbol{k}, \mathrm{i} \omega_{n}\right)$ in Fig. 16. Fig. 16(a) clearly shows the $d$-wave superconductivity with the node in the diagonal direction. It is a notable difference between the electron- and hole-doped cuprates that a wave function shows the sharp momentum dependence in the electron-doped case (Fig. 16(a)). This is because the quasi-particle and the Fermi surface are more clearly defined in the electron-doped cases. In the hole-doped cuprates, the many low energy states lie around $(\pi, 0)$ where the quasi-particles are broad. On the other hand, the Fermi surface is removed from $(\pi, 0)$ and the electron correlation is effectively weak in the electron-doped case. Therefore, the order parameter has a large value only in the vicinity of the Fermi surface.

The effectively weak correlation is consistent with the $T$-square resistivity in the electron-doped cuprates. 22 Our calculation shows the $\omega$-square dependence of the imaginary self-energy $\operatorname{Im} \Sigma_{\mathrm{F}}^{\mathrm{R}}(\boldsymbol{k}, \omega)$ in the electron-doped case. This is consistent with the experimental results 99 ) and implies the $T$-square resistivity. The incommensurate spin fluctuations have been regarded to give rise to the above momentum dependence of the wave function in the electron-doped case.102 However, it is actually not the main reason. Actually, the spin fluctuations are always commensurate in the electron-doped cuprates within our calculations.

Thus, the comprehensive understanding for the phase diagram including the particle-hole asymmetry further- 


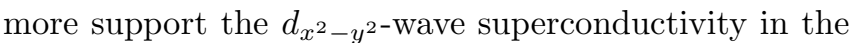
electron-doped cuprates.

(a) electron-doped

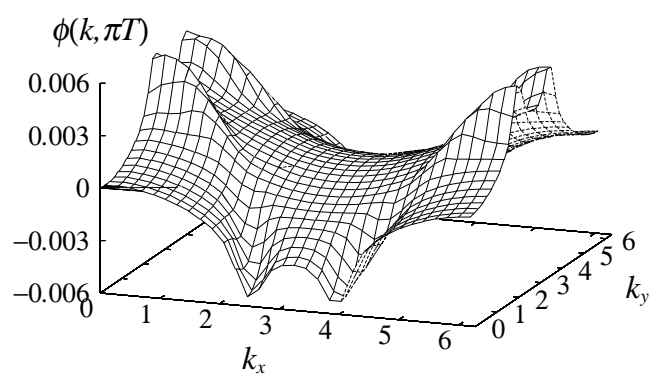

(b) hole-doped

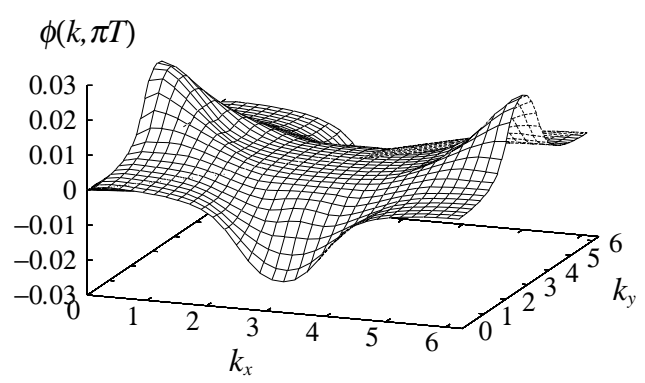

Fig. 16. The momentum dependence of the wave function $\phi\left(\boldsymbol{k}, \mathrm{i} \omega_{n}\right)$ at $\omega_{n}=\pi T$ (a) in the electron-doped cuprates $\left(\delta=-0.105\right.$ and $\left.T_{\mathrm{c}}=0.0040\right)$ and (b) in hole-doped cuprates $\left(\delta=0.093\right.$ and $\left.T_{\mathrm{c}}=0.0080\right)$.

From the above results, we expect that the pseudogap phenomena are not so remarkable in the electron-doped cuprates. Because of the low $T_{\mathrm{c}}$ and the weak electron correlation, the superconducting coupling $T_{\mathrm{c}} / \varepsilon_{\mathrm{F}}$ is small. Actually, our calculation gives the large TDGL parameter $b=30$ for the parameter set used in Fig. 16(a). Therefore, the superconducting fluctuations are not so significant as well as in the standard BCS superconductor.

The calculated self-energy shows only the weak effects which are the same order as those in over-doped cuprates. For example, the damping $-\operatorname{Im} \Sigma^{\mathrm{R}}(\boldsymbol{k}, 0)$ at $T=0.005$ and $\delta=-0.104$ is about $1 / 40$ of that for the under-doped case in Fig. 6. These results indicate that the pseudogap arising from the superconducting fluctuations is weak in the electron-doped cuprates, even if it is seen by some experiments. The results are consistent with the experimental results of ARPES99) and the neutron scattering 103 which do not show the pseudogap in the electron-doped cuprates. The weak effects give rise to the slight pseudogap in our calculation. However, the finite size effect seriously overestimates the effects of the superconducting fluctuations because of the large $b$. Therefore, the effects are precisely much weaker.

Thus, our calculation explains the doping dependence of High- $T_{\mathrm{c}}$ cuprates including the particle-hole asymmetry. The comprehensive understanding including both the hole- and electron-doped systems rather supports the pairing scenario for the pseudogap.

\section{§4. Self-Consistent Calculation}

In this section, we carry out the self-consistent calculation including the spin fluctuations, superconducting fluctuations and the single-particle properties. In the self-consistent calculation (We call the SC-FLEX+Tmatrix calculation), eqs. (2.3)-(2.8) and (3.5)-(3.9) are solved self-consistently where the fully dressed Green function $G\left(\boldsymbol{k}, \mathrm{i} \omega_{n}\right)=\left(\mathrm{i} \omega_{n}-\varepsilon_{\boldsymbol{k}}-\Sigma\left(\boldsymbol{k}, \mathrm{i} \omega_{n}\right)\right)^{-1}$ is used. By the self-consistent calculation, we calculate the critical temperature $T_{\mathrm{c}}$ reduced by the superconducting fuctuations. As is described in the previous papers, 19.33. there exists the singularity arising from the two-dimensionality. The singularity is actually removed by the weak three dimensionality which surely exists in the real systems. Therefore, we determine the critical temperature $T_{\mathrm{c}}$ as the temperature at which $\lambda(\mathbf{o}, 0)=0.98$ in order to avoid the unphysical singularity. This determination corresponds to that the dimensional crossover from the two-dimension to the threedimension occurs around $\lambda(\mathbf{o}, 0)=0.98$. The finite critical temperature is obtained by this operation. The method of the determination makes no significant difference on the following results. For example, the critical temperature is not so different (about $\sim 10 \%$ lower) even if we choose $\lambda(\mathbf{o}, 0)=0.99$ as the critical point. In particular, the similar doping dependence is obtained. Our choice of the parameter $\lambda(\mathbf{o}, 0)=0.98$ is owing to the reliability of the numerical calculation. Hereafter, we consider the hole-doped cases.

\subsection{Spin fluctuation and superconducting fluctuation}

First, we clarify the relation between the spin fluctuations and the superconducting fluctuations. The two fluctuations complicatedly couple to each other through the single particle properties. Here, we digest the important factors of the relations.

The superconducting fluctuations result from the spin fluctuations which act as the $d$-wave attractive interaction. The spin fluctuations give rise to the renormalization of the quasi-particles especially around $(\pi, 0)$. The renormalization reduces the effective Fermi energy $\varepsilon_{\mathrm{F}}$ for the $d$-wave superconductivity as well as the critical temperature $T_{\mathrm{c}}$. Therefore, the strong superconducting fluctuations are obtained at the reasonable temperature.

The effects of the superconducting fluctuations on the spin fluctuations have been investigated in $\S 3.4$. The superconducting fluctuations suppress the low frequency spin fluctuations and the anti-ferromagnetic order (see Fig. 11). A residual question is whether the feedback effects of the pseudogap on the spin fluctuations suppress the superconductivity or not.

In order to answer the question, we calculate the feedback effect on the critical temperature $T_{\mathrm{c}}$. We 
carry out the Modified FLEX (M-FLEX) calculation in which the fully dressed Green function is used only in eq. (2.6). In the other equations $G^{\mathrm{F}}\left(\boldsymbol{k}, \mathrm{i} \omega_{n}\right)=$ $\left(\mathrm{i} \omega_{n}-\varepsilon_{\boldsymbol{k}}-\Sigma_{\mathrm{F}}\left(\boldsymbol{k}, \mathrm{i} \omega_{n}\right)\right)^{-1}$ is used. Thus, the effects of the superconducting fluctuations on the spin fluctuations are included, however those on the single particle properties are not included. The eqs. (2.3)-(2.8) and (3.5)-(3.9) are solved self-consistently in the M-FLEX approximation. The results of the FLEX, M-FLEX and SC-FLEX+T-matrix calculations are shown in Table. I. Here, we determine the critical temperature by the condition $\lambda(\mathbf{o}, 0)=0.98$ for an equity.

\begin{tabular}{|c||c|c|c|l}
\hline & FLEX & M-FLEX & SC-FLEX+T-matrix & \\
\hline$T_{\mathrm{c}}$ & 0.0084 & 0.0098 & 0.0031 & \\
\hline$g \phi_{\max }^{2}$ & 24.18 & 14.23 & 20.1529 & \\
\hline$\gamma_{\mathrm{h}}$ & 0.06970 & 0.02747 & 0.04277 & \\
\hline$\gamma_{\mathrm{c}}$ & 0.00995 & 0.00971 & 0.00298 & \\
\hline & \multicolumn{4}{|c}{}
\end{tabular}

Table I. The comparison among the FLEX, M-FLEX and SCFLEX+T-matrix approximations. The critical temperature $T_{\mathrm{c}}$, the effective pairing interaction $g \phi_{\max }^{2}$, the damping at 'Hot spot' $\gamma_{\mathrm{h}}$ and that at 'Cold spot' $\gamma_{\mathrm{c}}$ at $T=T_{\mathrm{c}}$ are shown. The parameters are $U=1.6$ and $\delta=0.083 \sim 0.096$. Here, the self-energy in the M-FLEX approximation is that due to the spin fluctuations $\Sigma_{\mathrm{F}}^{\mathrm{R}}(\boldsymbol{k}, \omega)$.

In order to understand the results, it is important that the spin fluctuations have not only the paring effect but also the de-pairing effect. The former is from the relatively wide frequency region, and the latter is from the low frequency component. The pseudogap remarkably suppresses the low frequency component, however the total weight is not so reduced (Fig. 11). Thus, the pseudogap reduces the de-pairing effect rather than the pairing effect. Therefore, the higher critical temperature $T_{\mathrm{c}}=0.0098$ is obtained by the M-FLEX calculation where $T_{\mathrm{c}}=0.0084$ is obtained by the FLEX calculation. In other words, the feedback effects are advantageous to the superconductivity. Therefore, the feedback effects do not suppress the superconducting fluctuations and the pseudogap. Thus, only the properties of the low frequency component are not sufficient in order to understand the relation between the spin and superconducting fluctuations.

Needless to say, the pseudogap in the single particle properties reduces the critical temperature. The lower critical temperature $T_{\mathrm{c}}=0.0031$ is obtained by the SCFLEX+T-matrix calculation. The de-paring effect from the superconducting fluctuations is rather drastic than that from the spin fluctuations. (Therefore, the pseudogap is easily caused by the superconducting fluctuations.)

In order to make the above understanding clear, we show the quantities $g \phi_{\max }^{2}, \gamma_{\mathrm{h}}$ and $\gamma_{\mathrm{c}}$ in Table. I. Here, the quantity $\phi_{\max }$ is the maximum value of the order parameter $\phi\left(\boldsymbol{k}, \mathrm{i} \omega_{n}\right)$ and the quantity $g \phi_{\max }^{2}$ represents the strength of the pairing interaction. The damp- ing at the 'Hot spot' $\gamma_{\mathrm{h}}=-\operatorname{Im} \Sigma^{\mathrm{R}}\left(\boldsymbol{k}_{\mathrm{h}}, 0\right)$ at $T=T_{\mathrm{c}}$ represents the strength of the de-pairing effect. Here, $\boldsymbol{k}_{\mathrm{h}}=(0.98 \pi, 0.02 \pi)$. In should be noticed that the obtained value $\gamma_{\mathrm{h}}$ by the M-FLEX calculation has the minimum value in Table. I, although the temperature is highest. This fact shows that the de-pairing effect of the spin fluctuations are suppressed by the superconducting fluctuations.

The damping at the 'Cold spot' $\gamma=-\operatorname{Im} \Sigma^{\mathrm{R}}\left(\boldsymbol{k}_{\mathrm{c}}, 0\right)$ determines the in-plane transport 55, 56, 76. Here, $\boldsymbol{k}_{\mathrm{c}}=$ $(0.45 \pi, 0.42 \pi)$. The Table. I shows that the value $\gamma_{c}$ is not so reduced in the M-FLEX calculation from the FLEX calculation. Thus, the feedback effects through the spin fluctuations are small around the 'Cold spot'. Since the self-energy from the superconducting fluctuations $\Sigma_{\mathrm{S}}^{\mathrm{R}}\left(\boldsymbol{k}_{\mathrm{c}}, \omega\right)$ vanishes at the 'Cold spot', we can understand that the effect of the pseudogap on the in-plane transport is small 33, 6 ) as is observed experimentally. The slope of the T-linear resistivity is slightly reduced by the feedback effects. The anisotropy $\gamma_{\mathrm{h}} / \gamma_{\mathrm{c}}$ increases due to the superconducting fluctuations. This is important for the incoherent $c$-axis transport in the pseudogap state because the $c$-axis transport is determined by the 'Hot spot'.56, 76

\subsection{Results of the SC-FLEX+T-matrix calculation}

In this subsection, we show the results of the SCFLEX+T-matrix approximation. The qualitatively similar results to the FLEX+T-matrix approximation are obtained, although the effects of the superconducting fluctuations are reduced by the self-consistency. It is notable that the calculation treating a stronger electron-electron interaction $U$ is possible because the superconducting fluctuations suppress the anti-ferromagnetic order. Here, we choose the interaction $U=2.4$.

In Fig. 17, we show the self-energy calculated for the under-doped case $\delta=0.073$. The competition between the pseudogap and the Fermi liquid behavior is shown. The small imaginary part and the positive slope of the real part are obtained in the narrow region around the Fermi level. The anomalous features due to the superconducting fluctuations are shown in larger energy scale. These features are qualitatively the same as those obtained within the model with an attractive interaction.19. It should be noticed that these behaviors are given by the self-consistency between the superconducting fluctuations and the single particle properties. The necessity of the behaviors has been explained in ref. 19 .

The obtained spectral weight is shown in Fig. 18. The peak is shown near the Fermi level at the momentum around $(\pi, 0)$. This reflects the Fermi liquid behavior of the self-energy in Fig. 17. The typical three peak structure shown in the under-doped case $\delta=0.073$ (Fig. $18(\mathrm{a}))$ is a common feature to the results based on the model with an attractive interaction.19) It should be noticed that the weight around the Fermi level is remarkably reduced by the superconducting fluctuations which give the large imaginary part of the self-energy around the Fermi level. The spectral weight at the low energy region shifts to the high frequency region. The suppression 


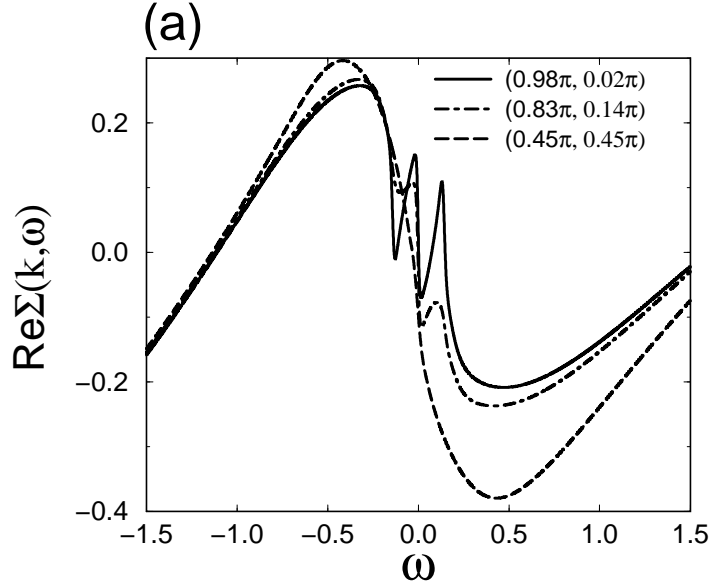

(b)

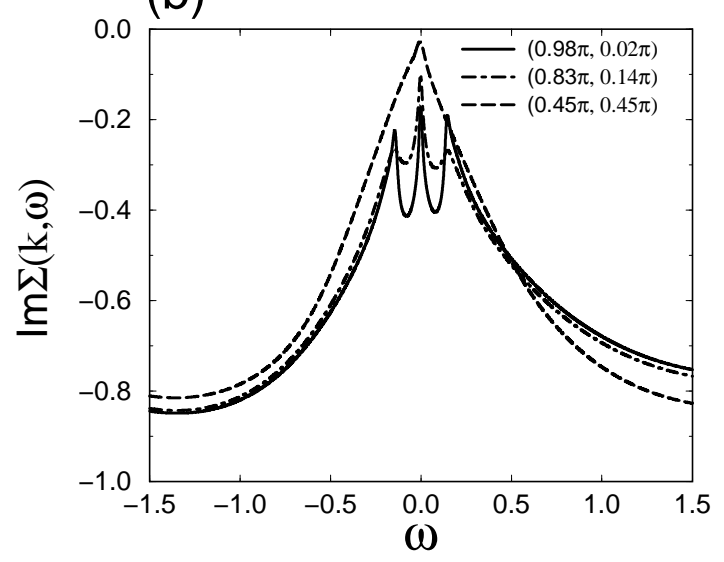

Fig. 17. The self-energy obtained by the SC-FLEX+T-matrix approximation. (a) The real part. (b) The imaginary part. Here, parameters are $U=2.4, \delta=0.073$ and $T=0.004$. The solid, dash-dotted and long-dashed lines are correspond to $\left(\frac{63}{64} \pi, \frac{1}{64} \pi\right)$, $\left(\frac{53}{64} \pi, \frac{9}{64} \pi\right)$ and $\left(\frac{29}{64} \pi, \frac{29}{64} \pi\right)$, respectively.

of the spectral weight near the Fermi level corresponds to the pseudogap.

The small weight near the Fermi level may not be observed by ARPES because the weight is so small. The ARPES measurements have their resolving power about the momentum and the energy. The thermal broadening arising from the Fermi distribution function $f(\omega)$ affects the ARPES furthermore. Anyway, the suppression of the spectral weight near the Fermi surface is a reliable result and consistent with the calculated result.

As is shown in the inset of Fig. 18(a), the usual single peak structure is obtained by neglecting the self-energy due to the superconducting fluctuations $\Sigma_{\mathrm{S}}\left(\boldsymbol{k}, \mathrm{i} \omega_{n}\right)$, namely $G\left(\boldsymbol{k}, \mathrm{i} \omega_{n}\right)=\left(\mathrm{i} \omega_{n}-\varepsilon_{\boldsymbol{k}}-\Sigma_{\mathrm{F}}\left(\boldsymbol{k}, \mathrm{i} \omega_{n}\right)\right)^{-1}$. The spectral weight is recovered and the three peak structure vanishes when the momentum leaves $(\pi, 0)$ along the Fermi surface. Similarly, the effects of the superconducting fluctuations are reduced by the hole-doping (Fig. $18(\mathrm{~b}))$. The single peak structure is obtained in the overdoped case $\delta=0.165$ (the inset in Fig. 18(b)). In other words, the pseudogap is reduced by the hole-doping because the superconducting fluctuations are suppressed.
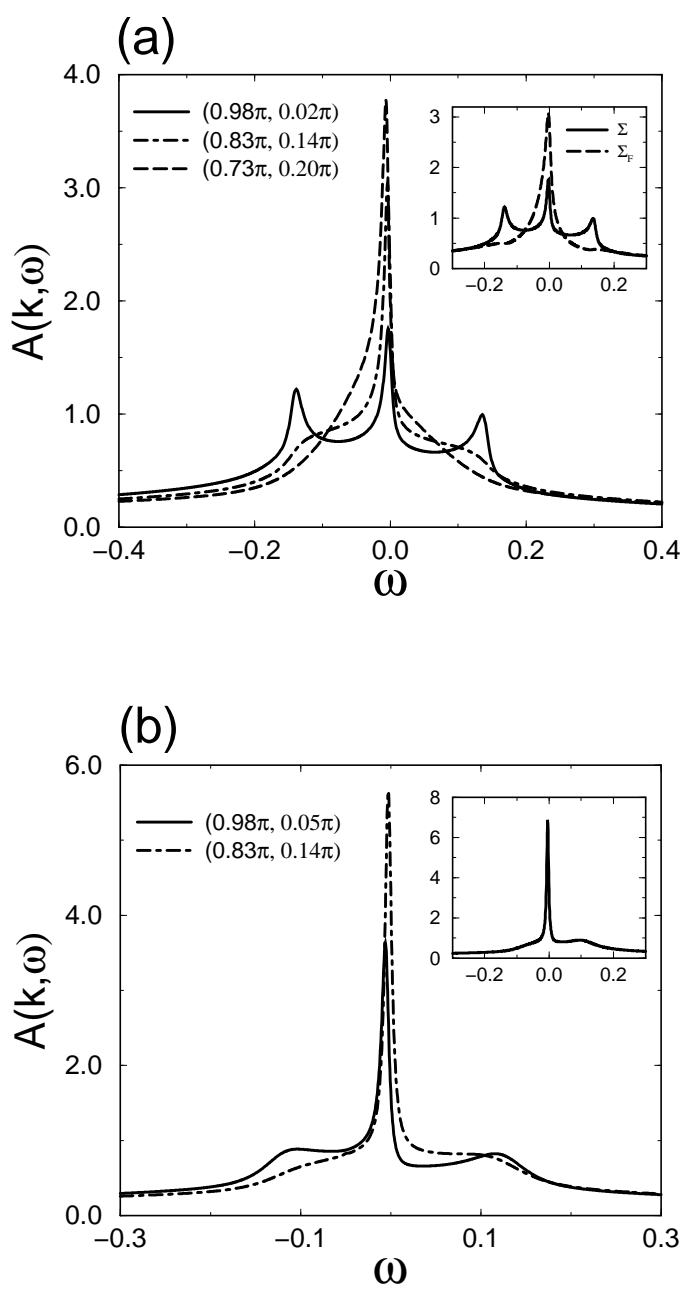

Fig. 18. The single particle spectral weight obtained by the SCFLEX+T-matrix approximation. (a) The under-doped case, $\delta=$ 0.073 and $T=0.004$. Here, $T_{\mathrm{c}}=0.0032$. The solid, dash-dotted and long-dashed lines are correspond to $\left(\frac{63}{64} \pi, \frac{1}{64} \pi\right),\left(\frac{53}{64} \pi, \frac{9}{64} \pi\right)$ and $\left(\frac{47}{64} \pi, \frac{13}{64} \pi\right)$, respectively. In the inset, the solid line shows the same result as in the main figure. The long-dashed lines show the result by neglecting the effects of the superconducting fluctuations $\Sigma_{\mathrm{S}}\left(\boldsymbol{k}, \mathrm{i} \omega_{n}\right)$. (b) The optimally-doped case, $\delta=0.119$ and $T=0.004$. Here, $T_{\mathrm{c}}=0.0035$. The solid and dash-dotted lines correspond to $\left(\frac{63}{64} \pi, \frac{3}{64} \pi\right)$ and $\left(\frac{53}{64} \pi, \frac{9}{64} \pi\right)$, respectively. The inset shows the result for the over-doped case, $\delta=0.165, T=$ 0.0035 and $k=\left(\frac{63}{64} \pi, \frac{5}{64} \pi\right)$.

The above effect of the superconducting fluctuations becomes more clear by showing the DOS in Fig. 19. The DOS near the Fermi level is reduced by the superconducting fluctuations and the gap structure appears in the under-doped case (solid line). It is confirmed by showing the result in which $\Sigma_{\mathrm{S}}\left(\boldsymbol{k}, \mathrm{i} \omega_{n}\right)$ is neglected (longdashed line) that the pseudogap in the DOS is caused by the superconducting fluctuations. The pseudogap is suppressed by the hole-doping, similarly (dash-dotted line). Thus, the pseudogap is properly obtained by the selfconsistent calculation including their doping dependence. 


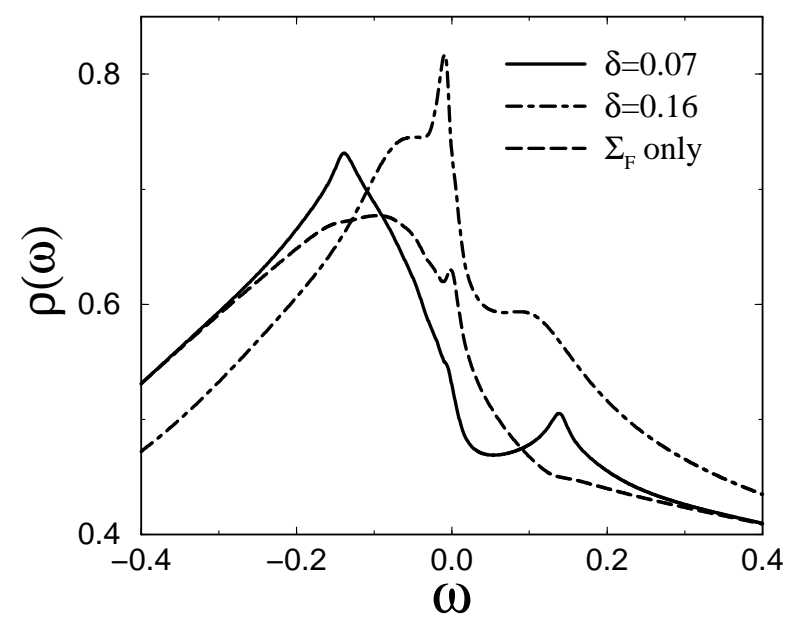

Fig. 19. The DOS obtained by the SC-FLEX+T-matrix approximation. The solid and dash-dotted lines show the under-doped case $(\delta=0.073$ and $T=0.004)$ and the over-doped case $(\delta=0.165$ and $T=0.0035)$, respectively. The long-dashed line shows the result for the under-doped case obtained by neglecting the self-energy $\Sigma_{\mathrm{S}}\left(\boldsymbol{k}, \mathrm{i} \omega_{n}\right)$.

At last, we show the obtained phase diagram in Fig. 20. The superconducting critical temperature $T_{\mathrm{c}}$ suppressed by the superconducting fluctuations is calculated by the self-consistent calculation. It should be noticed that the suppression of $T_{\mathrm{c}}$ from the mean field value becomes remarkable with under-doping. This is a natural result because the superconducting fluctuations become strong with under-doping. In other words, the pseudogap develops with under-doping and the reduced DOS gives the reduced critical temperature. An important result is the following; The critical temperature has the maximum value at $\delta \sim 0.11$ and decreases with under-doping in the SC-FLEX+T-matrix calculation for $U=2.4$, whereas $T_{\mathrm{c}}$ goes on increasing in the FLEX calculation. In other words, the mean field critical temperature $T_{\mathrm{c}}^{\mathrm{MF}}$ develops with under-doping. However, the decreasing $T_{\mathrm{c}}$ in the under-doped region is obtained by considering the superconducting fluctuations.

It is notable to write again that the strength of the superconducting coupling is indicated by the ratio $T_{\mathrm{c}}^{\mathrm{MF}} / \varepsilon_{\mathrm{F}}$, and not by $T_{\mathrm{c}}$. Since the effective Fermi energy $\varepsilon_{\mathrm{F}}$ decreases, the superconducting coupling becomes strong with under-doping in spite of the decreasing $T_{\mathrm{c}}$.

We can see from Fig. 20 that the critical temperature for $U=1.6$ does not decrease with under-doping even in the SC-FLEX+T-matrix calculation. Thus, the strong renormalization of the quasi-particles due to the strong electron correlation plays an important role for describing the under-doped cuprates. The other important thing is that we can treat the strong correlation by considering the superconducting fluctuations which suppresses the anti-ferromagnetic order. If neglecting the superconducting fluctuations, the system rapidly approaches the magnetic order. Because the magnetic or-

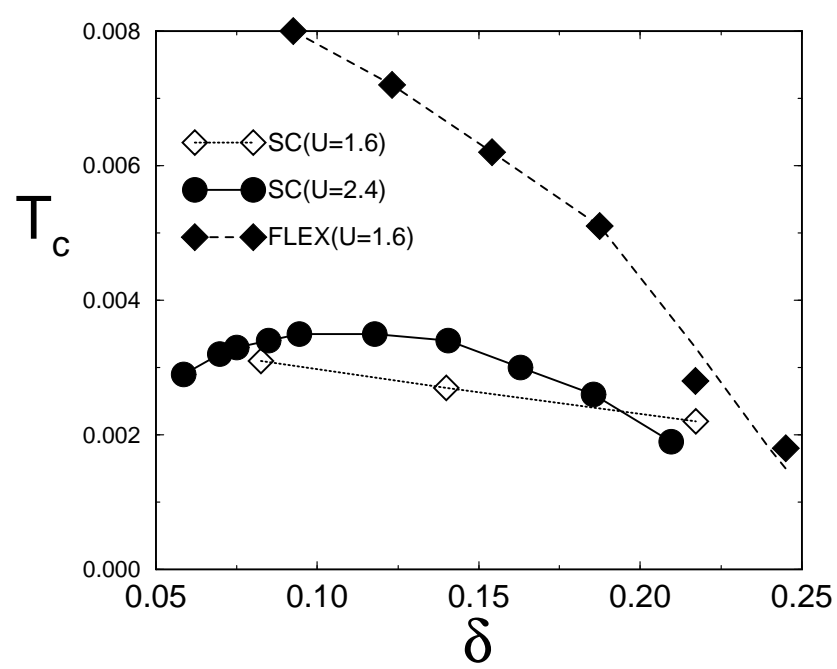

Fig. 20. The phase diagram obtained by the FLEX and the SCFLEX+T-matrix approximations. The closed circles show the results of the SC-FLEX+T-matrix approximation for $U=2.4$. The closed and open diamonds show the results of the FLEX and the SC-FLEX+T-matrix approximations for $U=1.6$.

der is suppressed by the superconducting fluctuations, the situation with the strong anti-ferromagnetic spin fluctuations persists in the under-doped region. This fact also contributes to the phase diagram shown in Fig 20.

The enhancement of the electron correlation effects with under-doping is necessary in order to obtain the phase diagram in which the critical temperature $T_{\mathrm{c}}$ decreases with under-doping. This doping dependence is probably underestimated in the FLEX approximation. The limitation of our calculation mainly arises from this fact. However, the doping dependence is partially included even in the FLEX approximation. Therefore, we can show the under-doped region in Fig. 20 although the decrease of the critical temperature $T_{\mathrm{c}}$ is not sufficient. This fact implies that the more sophisticated calculation which describes the stronger renormalization will gives the more decrease of the critical temperature $T_{\mathrm{c}}$. Anyway, the superconducting fluctuations play an important role in describing the under-doped region in the phase diagram.

\section{$\S 5$. Conclusion and Discussion}

In this paper, we have investigated the pseudogap phenomena starting from the Hubbard model. The superconducting fluctuations have been derived microscopically from the electron-electron correlation. We have succeeded in deriving the pseudogap phenomena by using the microscopic calculation.

The renormalized quasi-particles and the pairing interaction via the anti-ferromagnetic spin fluctuations are calculated by the FLEX approximation. The antiferromagnetic spin fluctuations give rise to the strong renormalization around the 'Hot spot'. Therefore, the effective Fermi energy $\varepsilon_{\mathrm{F}}$ for the $d$-wave symmetry is remarkably renormalized. Because of the high critical 
temperature $T_{\mathrm{c}}$ and the renormalized Fermi energy $\varepsilon_{\mathrm{F}}$, the superconducting coupling $T_{\mathrm{c}} / \varepsilon_{\mathrm{F}}$ becomes strong in the under-doped region. As a result, the pseudogap phenomena occurs owing to the self-energy correction by the strong superconducting fluctuations. The results support the scenario which is described within the model with a $d$-wave attractive interaction 19, 31, 32, 33, 34)

An important progress is that the pseudogap is described as a phenomenon near the Fermi surface. Because the obtained pairing interaction affects only the quasi-particles near the Fermi surface, it is naturally described that the superconducting fluctuations and the pseudogap have the small energy scale compared with the electron correlation. These results support the picture in which the pairing interaction becomes effectively strong for the renormalized quasi-particles.

Moreover, it should be emphasized that the calculation in this paper properly describes the doping dependence of the pseudogap phenomena. The superconducting coupling becomes weak with increasing the holedoping, since the Fermi energy increases and the critical temperature decreases. Therefore, the superconducting fluctuations become insignificant with hole-doping. The obtained doping dependence of the TDGL parameter $b$ has confirmed the scenario and is consistent with the the magnetic field dependence of the pseudogap phenomena. 34 )

The application of the calculation to the electrondoped case gives a consistent understanding with the experimental results.92, 97, 98, 99, 100, 103) We have obtained the $d_{x^{2}-y^{2}}$-wave superconductivity with low critical temperatures within the narrow doping range. It is shown that the superconducting fluctuations are weak and the pseudogap is not observed in the electron-doped case. The difference from the hole-doped case is derived from the particle-hole asymmetry of the band structure. The comprehensive understanding including the particle-hole asymmetry furthermore supports our scenario for High$T_{\mathrm{c}}$ cuprates.

The pseudogap in the magnetic properties has been investigated and explained properly. We have calculated the quantities observed by the NMR and the neutron scattering. The obtained results have naturally explained the characteristics of each quantities in the pseudogap state which are subtly different from each other.

We have clarified the relation between the spin fluctuations and the superconducting fluctuations which are complicatedly connected with each other. It is shown that the feedback effects of the superconducting fluctuations on the spin fluctuations enhance the superconductivity. Therefore, the feedback effect does not suppress the superconducting fluctuations.

Of course, the superconducting fluctuations themselves reduce the critical temperature. We have calculated the reduced critical temperature by the selfconsistent calculation including the single particle properties and the spin- and superconducting fluctuations. Qualitatively the similar results for the pseudogap have been obtained by the self-consistent calculation. The calculated critical temperature shows the maximum near $\delta \sim 0.11$, and decreases with under-doping, although it keeps increasing in the FLEX calculation. Thus, we have succeeded in describing the under-doped region by considering the strong superconducting fluctuations in the strongly correlated electron systems.

Finally, it should be stressed that the calculation in this paper starts from the Fermi liquid state and properly describes the High- $T_{\mathrm{c}}$ cuprates including the under-doped region. The comprehensive understanding of High- $T_{\mathrm{c}}$ cuprates has been obtained including their doping dependence from the over-doped region to the under-doped one.

The important and remained problem is the description of the under-doped limit. The calculation in this paper can not be applied to the limit because the FLEX approximation does not describe the Mott transition. The FLEX approximation probably underestimates the electron correlation and overestimates the anti-ferromagnetic correlation. Therefore, the antiferromagnetic correlation may be too strong in the under-doped region in this paper. The momentum independent component of the electron correlation is probably stronger and the anti-ferromagnetic correlation is less strong in the actual state. The calculation beyond the FLEX approximation is desirable in order to describe the under-doped region more precisely.

However, it is notable that the essence of the pseudogap phenomena in High- $T_{\mathrm{c}}$ cuprates has been explained in this paper on the basis of the strong superconducting fluctuations. It is a strong evidence for the pairing scenario that the sufficiently strong superconducting fluctuations and the pseudogap phenomena are derived from the Hubbard model.

\section{Acknowledgements}

The authors are grateful to Dr. S. Koikegami for providing the program of the FLEX calculation, and to Professor M. Ogata, Professor K. Yamada, Professor T. Takahashi and Mr. T. Sato for fruitful discussions. Numerical computation in this work was partly carried out at the Yukawa Institute Computer Facility. The present work was partly supported by a Grant-In-Aid for Scientific Research from the Ministry of Education, Science, Sports and Culture, Japan. One of the authors (Y.Y) has been supported by a Research Fellowships of the Japan Society for the Promotion of Science for Young Scientists.

[1] H. Yasuoka, T. Imai and T. Shimizu: Strong Correlation and Superconductivity (Springer Verlag, Berlin 1989), p. 254.

[2] For example, W. W. Warren, R. E. Walstedt, G. F. Brennert, R. J. Cava, R. Tycko, R. F. Bell and G. Dabbagh: Phys. Rev. Lett. 62 (1989) 1193; M. H. Julien, P. Carretta, M. Horvatić: Phys. Rev. Lett. 76 (1996) 4238.

[3] M. Takigawa, A. P. Reyes, P. C. Hammel, J. D. Thompson R. H. Heffner, Z. Fisk and K. C. Ott: Phys. Rev. B 43 (1991) 247; M. Takigawa: Phys. Rev. B 49 (1994) 4158.

[4] Y. Itoh, H. Yasuoka, Y. Fujiwara, Y. Ueda, T. Machi, I. Tomeno, K. Tai, N. Koshizuka and S. Tanaka: J. Phys. Soc. Jpn. 61 (1992) 1287; Y. Itoh, T. Machi, S. Adachi, A. Fukuoka, K. Tanabe and H. Yasuoka: J. Phys. Soc. Jpn. 67 (1998) 312

[5] K. Ishida, K. Yoshida, T. Mito, Y. Tokunaga, Y. Kitaoka, K. Asayama, Y. Nakayama, J. Shimoyama and K. Kishio: Phys. 
Rev. B 58 (1998) R5960.

[6] Y. Tokunaga, K. Ishida, K. Yoshida, T. Mito, Y. Kitaoka, Y. Nakayama, J. Shimoyama, K. Kishio, O. Narikiyo and K. Miyake: Physica B 284-288 (2000) 663.

[7] A. Goto and T. Shimizu: Phys. Rev. B 57 (1998) 7977.

[8] J. Rossat-Mignod, L. P. Regnault, C. Vettier, P. Burlet, J. Y. Henry and G. Lapertot: Physica B 169 (1991) 58; J. RossatMignod, L. P. Regnault, C. Vettier, P. Bourges, P. Burlet, J. Bossy, J. Y. Henry and G. Lapertot: Physica C 185\&189 (1991) 86.

[9] For example, T. Ito, K. Takenaka and S. Uchida: Phys. Rev. Lett. 70 (1993) 3995; K. Mizuhashi, K. Takenaka, Y. Fukuzumi and S. Uchida: Phys. Rev. B 52 (1995) R3884.

[10] M. Oda, K. Hoya, R. Kubota, C. Manabe, N. Momono, T. Nakano and M. Ido: Physica C 281 (1997) 135.

[11] For example, C. C. Homes, T. Timusk, R. Liang, D. A. Bonn and W. H. Hardy: Phys. Rev. Lett. 71 (1993) 1645; D. N. Basov, R. Liang, B. Dabrowski, D. A. Bonn, W. N. Hardy and T. Timusk: Phys. Rev. Lett. 77 (1996) 4090; S. Tajima, J. Schützmann, S. Miyamoto, I. Terasaki, Y. Sato and R. Hauff: Phys. Rev. B 55 (1997) 6051.

[12] J. W. Loram, K. A. Mirza, J. M. Wade, J. R. Cooper and W. Y. Liang: Physica C 235\&240 (1994) 134; N. Momono, T. Matsuzaki, T. Nagata, M. Oda and M. Ido: to be published in J. Low. Temp. Phys.

[13] Ch. Renner, B. Revaz, J.-Y. Genoud, K. Kadowaki and $\varnothing$. Fischer: Phys. Rev. Lett. 80 (1998) 149.

[14] H. Ding, T. Yokoya, J. C. Campuzano, T. Takahashi, M. Randeria, M. R. Norman, T. Mochiku, K. Kadowaki and J. Giapintzakis: Nature. 382 (1996) 51; A. G. Loeser, Z. X. Shen, D. S. Dessau, D. S. Marshall, C. H. Park, P. Fournier and A. Kapitulnik: Science. 273 (1996) 325; M. R. Norman, H. Ding, M. Randeria, J. C. Campuzano, T. Yokoya, T. Takeuchi, T. Takahashi, T. Mochiku, K. Kadowaki, P. Guptasarma and D. G. Hinks: Nature. 392 (1998) 157.

[15] T, Timusk and B. Statt: Rep. Prog. Phys. 62 (1999) 61.

[16] H. Fukuyama: Prog. Theor. Phys. Suppl. 108 (1992) 287; T. Tanamoto, H. Kohno and H. Fukuyama: J. Phys. Soc. Jpn 63 (1994) 2739.

[17] D. Pines: Z. Phys. B 103 (1997) 129 and references there in

[18] A. Kampf and J. R. Schrieffer: Phys. Rev. B 41 (1990) 6399; T. Dahm and L. Tewordt: Phys. Rev. B. 52 (1995) 1297; A. V. Chubukov, D. K. Morr and K. A. Shakhnovich: Philos. Mag. B 74 (1996) 563; A. V. Chubukov and J. Schmalian: Phys. Rev. B 57 (1998) 11085.

[19] Y. Yanase and K. Yamada: J. Phys. Soc. Jpn. 68 (1999) 2999.

[20] C. A. R. Sá de Melo, M. Randeria and J. R. Engelbrecht: Phys. Rev. Lett. 71 (1993) 3202; M. Randeria: Bose-Einstein Condensation ed. A. Griffin, D. Snoke and S. Stringari (Cambridge. Cambridge University Press, 1994); M. Randeria: condmat/9710223 and references there in.

[21] V. J. Emery and S. A. Kivelson: Phys. Rev. Lett. 74 (1995) 3253.

[22] M. Franz and A. J. Millis: Phys. Rev. B 58 (1998) 14572.

[23] H-J. Kwon and A. T. Dorsey: Phys. Rev. B 59 (1999) 6438.

[24] R. Haussmann: Phys. Rev. B 49 (1994) 12975.

[25] S. Stintzing and W. Zwerger: Phys. Rev. B 56 (1997) 9004.

[26] S. Koikegami and K. Yamada: J. Phys. Soc. Jpn. 67 (1998) 1114.

[27] A. Kobayashi, A. Tsuruta, T. Matsuura and Y. Kuroda: J. Phys. Soc. Jpn. 67 (1998) 2626.

[28] V. B. Geshkenbein, L. B. Ioffe and A. I. Larkin: Phys. Rev. B 55 (1997) 3173.

[29] B. Jankó, J. Maly and K. Levin: Phys. Rev. B 56 (1997) 11407; J. Maly, B. Jankó and K. Levin: Physica C 321 (1999) 113.

[30] Q. Chen, I. Kosztin, B. Jank, and K. Levin: Phys. Rev. Lett. 81 (1998) 4708; Q. Chen, I. Kosztin, and K. Levin: Phys. Rev. Lett. 85 (2000) 2801.

[31] T. Jujo and K. Yamada: J. Phys. Soc. Jpn. 68 (1999) 2198.

[32] T. Jujo, Y. Yanase and K. Yamada: J. Phys. Soc. Jpn. 69 (2000) 2240.
[33] Y. Yanase, T. Jujo and K. Yamada: J. Phys. Soc. Jpn. 69 (2000) 3664.

[34] Y. Yanase and K. Yamada: J. Phys. Soc. Jpn. 69 (2000) 2209.

[35] R. Micnas, M. H. Pedersen, S. Schafroth, T. Schneider, J. J. Rodríguez-Núñez and H. Beck: Phys. Rev. B 52 (1995) 16223.

[36] J. R. Engelbrecht, A. Nazarenko, M. Randeria and E. Dagotto: Phys. Rev. B 57 (1998) 13406.

[37] A. Kobayashi, A. Tsuruta, T. Matsuura and Y. Kuroda: J. Phys. Soc. Jpn. 68 (1999) 2506.

[38] S. Onoda and M. Imada: J. Phys. Soc. Jpn. 68 (1999) 2762; ibid. 69 (2000) 312; ibid. 69 Suppl. B (2000) 32.

[39] S. Koikegami and K. Yamada: J. Phys. Soc. Jpn. 69 (2000) 768; J. Phys. Soc. Jpn. 69 (2000) 1950.

[40] T. Dahm, D. Manske and L. Tewordt: Phys. Rev. B 55 (1997) 15274

[41] A. Perali, C. Castellani, C. Di Castro, M Grilli, E. Piegari and A. A. Varlamov: Phys. Rev. B 62 (2000)R9295.

[42] D. Rohe and W. Metzner: cond-mat/0011500.

[43] Y. J. Uemura et al.: Phys. Rev. Lett. 62 (1989) 2317.

[44] P. Nozières and S. Schmitt-Rink: J. Low Temp. Phys. 59 (1985) 195; A. J. Leggett: Modern Trends in the Theory of Condensed Matter ed. A. Pekalski and R. Przystawa (SpringerVerlag, Berlin, 1980).

[45] S. Schmitt-Rink, C. M. Varma and A. E. Ruckenstein: Phys. Rev. Lett. 63 (1989) 445; A. Tokumitu, K. Miyake and K. Yamada: Prog. Theor. Phys. Suppl. 106 (1991) 63.

[46] T. Nagaoka, Y. Matsuda, H. Obara, A. Sawa, T. Terashima, I. Chong, M. Takano and M. Suzuki: Phys. Rev. Lett. 80 (1998) 3594; A. G. Aronov and A. B. Rapoport: Mod. Phys. Lett. B 6 (1992) 1083; A. G. Aronov, S. Hikami and A. I. Larkin; Phys. Rev. B 51 (1995) 3880.

[47] L. G. Aslamazov and A. I. Larkin: Fiz. Tverd. Tela. 10 (1968) 1104. [Sov. Phys. Solid State 10 (1968) 875.]

[48] K. Maki: Prog. Theor. Phys 40 (1968) 193.; R. S. Thompson: Phys. Rev. B 1 (1970) 327.

[49] G-q. Zheng, W. G. Clark, Y. Kitaoka, K. Asayama, K. Kodama, P. Kuhns and W. G. Moulton: Phys. Rev. B 60 (1999) R9947.

[50] K. Gorny, O. M. Vyaselev, J. A. Martindale, V. A. Nandor, C. H. Pennington, P. C. Hammel, W. L. Hults, J. L. Smith, P. L. Kuhns, A. P. Reyes and W. G. Moulton: Phys. Rev. Lett. 82 (1999) 177.

[51] M. Eschrig, D. Rainer and J. A. Sauls: Phys. Rev. B 59 (1999) 12095.

[52] G-q. Zheng, H. Ozaki, W. G. Clark, Y. Kitaoka, P. Kuhns, A. P. Reyes, W. G. Moulton, T. Kondo, Y. Shimakawa and Y. Kubo: Phys. Rev. Lett. 85 (2000) 405.

[53] T. Moriya, Y. Takahashi and K. Ueda: J. Phys. Soc. Jpn 59 (1990) 2905; K. Ueda, T. Moriya and Y. Takahashi: J. Phys. Chem. Solids 53 (1992) 1515.

[54] P. Monthoux, A. V. Balatsky and D. Pines: Phys. Rev. B 46 (1992) 14803

[55] B. P. Stojković and D. Pines: Phys. Rev. Lett. 76 (1996) 811; B. P. Stojković and D. Pines: Phys. Rev. B 55 (1997) 8576.

[56] Y. Yanase and K. Yamada: J. Phys. Soc. Jpn 68 (1999) 548.

[57] N. E. Bickers, D. J. Scalapino and S. R. White: Phys. Rev. Lett. 62 (1989) 961; N. E. Bickers and D. J. Scalapino: Ann. Phys. (N.Y.) 193 (1989) 206.

[58] G. Baym and L. P. Kadanoff: Phys. Rev. 124 (1961) 287.

[59] T. Moriya: Spin Fluctuations in Itinerant Electron Magnetism (Springer-Verlag, 1985).

[60] T. Moriya and K. Ueda: Adv. Phys. 49 (2000) 555.

[61] P. Monthoux and D. J. Scalapino: Phys. Rev. Lett. 72 (1994) 1874.

[62] C.-H. Pao and N. E. Bickers: Phys. Rev. Lett. 72 (1994) 1870; Phys. Rev. B 51 (1995) 16310.

[63] T. Dahm and L. Tewordt: Phys. Rev. Lett. 74 (1995) 793; Phys. Rev. B 52 (1995) 1297.

[64] M. Langer, J. Schmalian, S. Grabowski and K. H. Bennemann: Phys. Rev. Lett. 75 (1995) 4508.

[65] J. J. Deisz, D. W. Hess and J. W. Serene: Phys. Rev. Lett. 76 (1996) 1312. 
[66] S. Koikegami, S. Fujimoto and K. Yamada: J. Phys. Soc. Jpn 66 (1997) 1438.

[67] T. Takimoto and T. Moriya: J. Phys. Soc. Jpn 66 (1997) 2459.

[68] H. Kino and H. Kontani: J. Phys. Soc. Jpn. 67 (1998) 3691.

[69] H. Kondo and T. Moriya: J. Phys. Soc. Jpn. 67 (1998) 3695.

[70] J. Schmalian: Phys. Rev. Lett. 81 (1998) 4232.

[71] H. Kontani and K. Ueda: Phys. Rev. Lett. 80 (1998) 5619.

[72] H. Kontani, K. Kanki and K. Ueda: Phys. Rev. B 59 (1999) 14723.

[73] K. Kanki and H. Kontani: J. Phys. Soc. Jpn 68 (1999) 1614.

[74] T. Takimoto and T. Moriya: J. Phys. Soc. Jpn 67 (1998) 3570 .

[75] K. Miyake and O. Narikiyo: J. Phys. Soc. Jpn 63 (1994) 3821.

[76] L. B. Ioffe and A. J. Millis: Phys. Rev. B 58 (1998) 11631; V. B. Geshkenbein, L. B. Ioffe and A. J. Millis: Phys. Rev. Lett. 80 (1998) 5778.

[77] H. Ebisawa and H. Fukuyama: Prog. Theor. Phys 46 (1971) 1042.

[78] V. Barzykin and D. Pines: Phys. Rev. B 52 (1995) 13585.

[79] N. Bulut and D. J. Scalapino: Phys. Rev. Lett. 67 (1991) 2898.

[80] M. Oda, H. Matsuki and M. Ido: Solid State Commun. 74 (1990) 1321.

[81] P. Dai, H. A. Mook, and F. Doğgan: Phys. Rev. Lett. 80 (1998) 1738; M. Arai, T. Nishijima, Y. Endoh, T. Egami, S. Tajima, K. Tomimoto, Y. Shiohara, M. Takahashi, A. Garrett and S. M. Bennington: Phys. Rev. Lett. 83 (1999) 608.

[82] J. M. Tranquada, B. J. Sternlieb, J. D. Axe, Y. Nakamura and S. Uchida: Nature 375 (1995) 561; J. M. Tranquada, J. D. Axe, N. Ichikawa, Y. Nakamura, S. Uchida and B. Nachumi: Phys. Rev. B 54 (1996) 7489.

[83] J. Zaanen and A. M. Oleś: Ann. Phys. 5 (1996) 224.

[84] V. J. Emery, S. A. Kivelson and O. Zachar: Phys. Rev. B. 56 (1997) 6120

[85] S. R. White and D. J. Scalapino: Phys. Rev. Lett. 80 (1998) 1272 .

[86] T. Mizokawa and A. Fujimori: Phys. Rev. Lett. 80 (1998) 1320.

[87] H. Yamase, H. Kohno, H. Fukuyama and M. Ogata: J. Phys. Soc. Jpn 68 (1999) 1082.

[88] K. Machida and M. Ichioka: J. Phys. Soc. Jpn 68 (1999) 2168; M. Ichioka and K. Machida: J. Phys. Soc. Jpn 68 (1999) 4020.

[89] A. Himeda, T. Kato and M. Ogata: preprint.

[90] K. Yamada, C. H. Lee, K. Kurahashi, J. Wada, S. Wakimoto, S. Ueki, H. Kimura, Y. Endoh, S. Hosoya, G. Shirane, R. J. Birgeneau, M. Greven, M. A. Kastner, and Y. J. Kim: Phys. Rev. B. 57 (1998) 6165.

[91] K. Kuroki, R. Arita and H. Aoki: Phys. Rev. B. 60 (1999) 9850.

[92] Y. Tokura, H. Takagi and S. Uchida: Nature. 337 (1989) 345; H. Takagi, S. Uchida and Y. Tokura: Phys. Rev. Lett. 62 (1989) 1197

[93] D. H. Wu, J. Mao, S. N. Mao, J. L. Peng, X. X. Xi, T. Venkatesan, R. L. Greene, and S. M. Anlage: Phys. Rev. Lett. 70 (1993) 85; A. Andreone, A. Cassinese, A. Di Chiara, R. Vaglio, A. Gupta, and E. Sarnelli: Phys. Rev. B. 49 (1994) 6392; C. W. Schneider, Z. H. Barber, J. E. Evetts, S. N. Mao, X. X. Xi and T. Venkatesan: Physica C 233 (1994) 77; S. M. Anlage, D.-H. Wu, J. Mao, S. N. Mao, X. X. Xi, T. Venkatesan, J. L. Peng, and R. L. Greene: Phys. Rev. B. 50 (1994) 523; L. Alff, S. Meyer, S. Kleefisch, U. Schoop, A. Marx, H. Sato, M. Naito, and R. Gross: Phys. Rev. Lett. 83 (1999) 2644.

[94] S. Kashiwaya, T. Ito, K. Oka, S. Ueno, H. Takashima, M. Koyanagi, Y. Tanaka, and K. Kajimura: Phys. Rev. B. 57 (1998) 8680; L. Alff, A. Beck, R. Gross, A. Marx, S. Kleefisch, Th. Bauch, H. Sato, M. Naito, and G. Koren: Phys. Rev. B. 58 (1998) 11197.

[95] Y. Tanaka and S. Kashiwaya: Phys. Rev. Lett. 74 (1995) 3451; S. Kashiwaya, Y. Tanaka, M. Koyanagi, H. Takashima and K. Kajimura: Phys. Rev. B. 51 (1995) 1350; S. Kashiwaya, Y. Tanaka, M. Koyanagi and K. Kajimura: Phys. Rev. B. 53 (1996) 2667.
[96] M. Ogata: J. Phys. Soc. Jpn 66 (1997) 3375; K. Kuroki and H. Aoki: J. Phys. Soc. Jpn 67 (1998) 1533.

[97] J. D. Kokales, P. Fournier, L. V. Mercaldo, V. V. Talanov, R L. Greene, S. M. Anlage: preprint. Phys. Rev. Lett. 85 (2000) 3696; R. Prozorov, R. W. Giannetta, P. Fournier, R. L. Greene: Rev. Lett. 85 (2000) 3700.

[98] F. Hayashi, E. Ueda, M. Sato, K. Kurahashi and K. Yamada: J. Phys. Soc. Jpn 67 (1998) 3234.

[99] T. Takahashi and T. Sato: private communications.

[100] C. C. Tsuei and J. R. Kirtley: Phys. Rev. Lett. 85 (2000) 182 .

[101] H. Kondo and T Moriya: J. Phys. Soc. Jpn 68 (1999) 3170.

[102] D. Manske, I. Eremin and K. H. Bennemann: Phys. Rev. B. $62(2000) 13922$.

[103] K. Yamada: private communications. 